\title{
Modelling of residually stressed materials with application to AAA
}

\author{
T. Ahamed ${ }^{\mathrm{a}}$, L. Dorfmann ${ }^{\mathrm{a}, *}$, R.W. Ogden ${ }^{\mathrm{b}}$ \\ ${ }^{a}$ Department of Civil and Environmental Engineering \\ Tufts University, Medford MA, 02155 \\ ${ }^{b}$ School of Mathematics and Statistics, University of Glasgow, UK
}

\begin{abstract}
Residual stresses are generated in living tissues by processes of growth and adaptation and they significantly influence the mechanical behaviour of the tissues. Thus, to effectively model the elastic response of the tissues relative to a residually stressed configuration the residual stresses need to be incorporated into the constitutive equations. The purposes of this paper are (a) to summarise a general elastic constitutive formulation that includes residual stress, (b) to specify the tensors needed for the three-dimensional implementation of the theory in a nonlinear finite element code, and (c) to use the theory and its implementation to evaluate the wall stress distribution in an abdominal aortic aneurysm (AAA) using patient specific geometry and material model parameters. The considered material is anisotropic with two preferred directions indicating the orientation of the collagen fibres in the aortic tissue. The method described in this paper is general and can be used, by specifying appropriate energy functions, to investigate other residually stressed biological systems.
\end{abstract}

Keywords: residual stress, abdominal aortic aneurysm, AAA, finite element analysis

\footnotetext{
*Corresponding author

Email address: luis.dorfmann@tufts.edu; Tel.(617)-627-6137 (L. Dorfmann) $U R L:$ http://ase.tufts.edu/msml/ (L. Dorfmann)
} 


\section{Introduction}

Stresses that are present in a material body in the absence of any applied loads (either body forces or surface tractions) are referred to as residual stresses, as in the definition of Hoger (1985). The existence of residual stresses in plants, human tissues, insects and other animals has been the subject of many publications, including, for example, Nienhaus et al. (2009), Vandiver and Goriely (2009), Holzapfel and Ogden (2010) and Tierney et al. (2010). They are associated with tissue growth and remodelling and we refer to Taber (1995), Skalak et al. (1996), Yeni et al. (2002), Ambrosi et al. (2008), Olsson and Klarbring (2008), Vandiver and Goriely (2009) and Wells and Walter (2010), and to the references therein, for a detailed discussion of various aspects of the development of residual stresses due to morphological changes in the tissues.

That residual stresses are present in biological materials has been known for a long time, but their consequences for the behaviour of biological systems, from a biomechanics point of view, are yet to be fully appreciated. This has created considerable interest during the last few years and many valuable publications have resulted from attempts to understand the influence of residual stresses on the biomechanical behaviour of, in particular, artery walls and the heart.

To characterize the effect that residual stresses have on the behaviour of biological systems it is necessary to know their actual spatial distribution. Fung (1983) emphasized the influence of residual stresses in explaining the experimentally observed responses of tubular sections of arterial tissue, and he introduced the so-called opening angle experiment in order to quantify the magnitude of the residual stress (Chuong and Fung, 1986), although this technique gives only a crude measure of residual stresses. It was also used by Vaishnav and Vossoughi (1987) to estimate the residual stress distribution and the corresponding strains in bovine and porcine aortas. The opening angle test is essentially two dimensional and reveals only information about the radial and circumferential residual stresses in a cross section of an artery wall, although it is well known that axial residual stresses also exist. To account for the latter, a three-dimensional analytical method for determining the layer specific residual stress distribution using data from a human aorta was developed by Holzapfel and Ogden (2010).

Experimental techniques have been used to determine residual stresses indirectly by measuring the deformation associated with the relief of resid- 
ual stress by cutting tissue specimens. For example, Costa et al. (1997) determined the three-dimensional strain distribution in a canine left ventricle sample after cutting relative to the intact state and were able to infer information about the associated residual stresses that were present prior to the cut, although they did not quantify these. Similarly, Wang et al. (2015) measured strains in ocular tissues resulting from stress-relieving cuts. These authors refer to 'residual strains' associated with residual stresses. However, we emphasize that, unlike true (residual) stress, strain depends on the choice of reference configuration, and in the present work we consider strain to be measured from the residually stressed configuration (and therefore to vanish in that configuration). Thus, we do not associate a strain with a residual stress.

Fung (1983) and Chuong and Fung (1986) emphasized the need to include residual stresses in any analysis used to determine the actual stress distribution in the loaded material. With this in mind and using appropriate constitutive equations, Holzapfel et al. (2000) performed a detailed stress analysis of the arterial wall and compared the stress distribution with and without taking residual stress into account, therefore highlighting the significant difference that residual stresses can make. In general, analytical methods used to determine the arterial wall stress distributions assume, as in Holzapfel et al. (2000), that the artery is a circular tube of incompressible material, with constant cross section and wall thickness and subject to a constant pressure. Analytical methods must be replaced by a numerical approach if these calculations are to be applied to patient specific geometries.

Using a numerical approach in an analysis of the elasticity of the human left ventricle in diastole, Wang et al. (2014) adopted the Holzapfel and Ogden (2009) constitutive formulation modified to include residual stresses. Based on the analysis of Holzapfel and Ogden (2010), the effect of residual stresses was also incorporated into the finite element formulation of Pierce et al. (2015), which was used to determine the wall stress distribution of patientspecific abdominal aortic aneurysms (AAAs). For a review of the literature on modelling the mechanics of human abdominal aortas and AAAs we refer to Humphrey and Holzapfel (2012).

In the present paper we are also concerned with the application of an elasticity model that includes residual stresses to the computational analysis of the wall stress distribution in a patient specific AAA geometry when subject to a physiological pressure. We begin in Section 2 with an overview of the basic equations required to present a general elastic, invariant-based 
anisotropic constitutive formulation that includes residual stress. In Section 3 we develop a three-dimensional incremental formulation appropriate for the implementation of the theory in a nonlinear finite element code, including a suitable elasticity tensor. For numerical purposes the general constitutive formulation is specialized to a simple prototype model and a simple representation of the residual stress distribution is adopted.

As well as possessing anisotropy associated with residual stresses in its unloaded (reference) configuration, the considered material has anisotropy corresponding to two preferred directions that are identified with the orientations of two families of collagen fibres in the aortic tissue. In Section 4 we describe the patient specific finite element model, the experimentally determined material parameters, the fibre orientations and, in Section 5, the results of the finite element calculations. Section 6 contains some concluding remarks.

\section{Basic equations}

\subsection{Kinematics}

The region $\mathcal{B}_{\mathrm{r}}$ occupied by an unloaded, residually stressed deformable body is chosen as the reference configuration from which the deformation is measured. The location of a material particle in $\mathcal{B}_{\mathrm{r}}$ is identified by its position vector $\mathbf{X}$ relative to some fixed origin. Let $\mathcal{B}$ denote the configuration of the body after deformation from $\mathcal{B}_{\mathrm{r}}$, with $\mathbf{x}$ denoting the position vector occupied by the material particle which was at $\mathbf{X}$ in $\mathcal{B}_{\mathrm{r}}$. The deformation is described in terms of the vector function $\chi$ through $\mathbf{x}=\chi(\mathbf{X}), \mathbf{X} \in \mathcal{B}_{\mathrm{r}}$, and it is assumed that $\chi$ has sufficient regularity for the ensuing analysis.

The deformation gradient tensor $\mathbf{F}$ relative to the configuration $\mathcal{B}_{\mathrm{r}}$ is defined by

$$
\mathbf{F}=\operatorname{Grad} \boldsymbol{\chi}(\mathbf{X})=\operatorname{Grad} \mathbf{x}
$$

where Grad is the gradient operator with respect to $\mathbf{X}$, as distinct from grad, the usual gradient with respect to $\mathbf{x}$. The deformation gradient has Cartesian components $F_{i \alpha}=\partial x_{i} / \partial X_{\alpha}$, where $i, \alpha \in\{1,2,3\}$. Roman indices are associated with the current configuration $\mathcal{B}$ and Greek indices with the reference configuration $\mathcal{B}_{\mathrm{r}}$. We also use the standard notation $J$ to denote the determinant of $\mathbf{F}$ :

$$
J=\operatorname{det} \mathbf{F} \text {. }
$$


Note that $J$, which by convention is taken to be positive, relates an infinitesimal volume element $\mathrm{d} v$ in $\mathcal{B}$ to its counterpart $\mathrm{d} V$ in $\mathcal{B}_{\mathrm{r}}$ via $\mathrm{d} v=J \mathrm{~d} V$, a connection which will be used later.

Associated with $\mathbf{F}$ are the right and left Cauchy-Green deformation tensors defined, respectively, by

$$
\mathbf{C}=\mathbf{F}^{\mathrm{T}} \mathbf{F}, \quad \mathbf{B}=\mathbf{F F}^{\mathrm{T}}
$$

where ${ }^{\mathrm{T}}$ indicates the transpose of a second-order tensor. Of particular interest are the principal invariants of $\mathbf{C}$ (equivalently of $\mathbf{B}$ ) given by

$$
I_{1}=\operatorname{tr} \mathbf{C}, \quad I_{2}=\frac{1}{2}\left[I_{1}^{2}-\operatorname{tr}\left(\mathbf{C}^{2}\right)\right], \quad I_{3}=\operatorname{det} \mathbf{C} \equiv J^{2} .
$$

For an incompressible material $J=1$ and $I_{3}=1$, and we remark that the incompressibility idealization is often adopted for biological tissues.

For full details of the background on continuum kinematics and the associated theory of nonlinear elasticity we refer to Ogden (1997) and Holzapfel (2000).

\subsection{Structure tensors}

Suppose that in its reference configuration the material is endowed with a structure that is identified by the presence of preferred directions corresponding to unit vectors $\mathbf{M}(\mathbf{X})$ and $\mathbf{M}^{\prime}(\mathbf{X})$, typically associated with the mean directions of two families of collagen fibres which endow the material with anisotropic properties. From these preferred directions are defined the structure tensors $\mathbf{M} \otimes \mathbf{M}$ and $\mathbf{M}^{\prime} \otimes \mathbf{M}^{\prime}$. Following Spencer and Rivlin (1959) and Spencer (1971) we note that the integrity basis for three symmetric second-order tensors in three dimensions includes invariants, typically denoted $I_{4}, I_{5}, I_{6}, I_{7}$, that depend on $\mathbf{M} \otimes \mathbf{M}$ and $\mathbf{M}^{\prime} \otimes \mathbf{M}^{\prime}$ and defined by

$$
I_{4}=\mathbf{M} \cdot(\mathbf{C M}), I_{5}=\mathbf{M} \cdot\left(\mathbf{C}^{2} \mathbf{M}\right), I_{6}=\mathbf{M}^{\prime} \cdot\left(\mathbf{C M}^{\prime}\right), I_{7}=\mathbf{M}^{\prime} \cdot\left(\mathbf{C}^{2} \mathbf{M}^{\prime}\right)
$$

and additionally a coupling invariant, denoted $I_{8}$, that involves both fibre directions defined by $\mathbf{M} \cdot\left(\mathbf{C M}^{\prime}\right) \mathbf{M} \cdot \mathbf{M}^{\prime}$, which is independent of $I_{1}, \ldots, I_{7}$ if $\mathbf{M} \cdot \mathbf{M}^{\prime} \neq 0$. If $\mathbf{M} \cdot \mathbf{M}^{\prime}=0$ then it may alternatively be defined by $\left[\mathbf{M} \cdot\left(\mathbf{C} \mathbf{M}^{\prime}\right)\right]^{2}$, but in this case the latter can be expressed in terms of $I_{1}, \ldots, I_{7}$, specifically

$$
\left[\mathbf{M} \cdot\left(\mathbf{C M}^{\prime}\right)\right]^{2}=I_{2}+I_{4} I_{6}+I_{5}+I_{7}-I_{1}\left(I_{4}+I_{6}\right)
$$


as given in Merodio and Ogden (2006) for an incompressible material. The same formula applies for a compressible material with the appropriate definition of $I_{2}$.

In the reference configuration the above invariants reduce to

$$
I_{1}=I_{2}=3, \quad I_{3}=I_{4}=I_{5}=I_{6}=I_{7}=1, \quad I_{8}=\left(\mathbf{M} \cdot \mathbf{M}^{\prime}\right)^{2} .
$$

\subsection{Residual stress}

We now suppose that the residual stress tensor in the reference configuration $\mathcal{B}_{\mathrm{r}}$ is denoted by $\boldsymbol{\tau}$, and that there are no intrinsic couples in this configuration, so that $\boldsymbol{\tau}$ is symmetric and satisfies the equilibrium equation

$$
\operatorname{Div} \boldsymbol{\tau}=\mathbf{0}
$$

where Div denotes the divergence operator with respect to $\mathcal{B}_{\mathrm{r}}$. Since the reference configuration is unloaded there are no surface tractions applied to the boundary $\partial \mathcal{B}_{\mathrm{r}}$, so $\boldsymbol{\tau}$ must also satisfy the boundary condition

$$
\boldsymbol{\tau} \mathbf{N}=\mathbf{0} \quad \text { on } \partial \mathcal{B}_{\mathrm{r}}
$$

where $\mathbf{N}$ is the unit outward normal to the boundary $\partial \mathcal{B}_{\mathrm{r}}$ of $\mathcal{B}_{\mathrm{r}}$. Note that (8) and (9) imply that the residual stress in $\mathcal{B}_{\mathrm{r}}$ is non-uniform.

Associated with $\boldsymbol{\tau}$ are invariants analogous to those of $\mathbf{C}$. We denote these by $R_{1}, \ldots, R_{8}$ with the definitions

$$
\begin{gathered}
R_{1}=\operatorname{tr} \boldsymbol{\tau}, \quad R_{2}=\frac{1}{2}\left[R_{1}^{2}-\operatorname{tr}\left(\boldsymbol{\tau}^{2}\right)\right], \quad R_{3}=\operatorname{det} \boldsymbol{\tau} \\
R_{4}=\mathbf{M} \cdot(\boldsymbol{\tau} \mathbf{M}), R_{5}=\mathbf{M} \cdot\left(\boldsymbol{\tau}^{2} \mathbf{M}\right), R_{6}=\mathbf{M}^{\prime} \cdot\left(\boldsymbol{\tau} \mathbf{M}^{\prime}\right), R_{7}=\mathbf{M}^{\prime} \cdot\left(\boldsymbol{\tau}^{2} \mathbf{M}^{\prime}\right),
\end{gathered}
$$

and $R_{8}=\mathbf{M} \cdot\left(\boldsymbol{\tau} \mathbf{M}^{\prime}\right) \mathbf{M} \cdot \mathbf{M}^{\prime}$ or $\left[\mathbf{M} \cdot\left(\boldsymbol{\tau} \mathbf{M}^{\prime}\right)\right]^{2}$. Since these are independent of the deformation it will be convenient later to denote this set of invariants collectively as $R=\left\{R_{1}, R_{2}, \ldots, R_{8}\right\}$.

Next, we list the invariants that depend only on $\boldsymbol{\tau}$ and $\mathbf{C}$. There are just four independent such invariants, which we denote by $I_{9}, I_{10}, I_{11}, I_{12}$, these being defined by

$$
I_{9}=\operatorname{tr}(\mathbf{C} \boldsymbol{\tau}), \quad I_{10}=\operatorname{tr}\left(\mathbf{C} \boldsymbol{\tau}^{2}\right), \quad I_{11}=\operatorname{tr}\left(\mathbf{C}^{2} \boldsymbol{\tau}\right), \quad I_{12}=\operatorname{tr}\left(\mathbf{C}^{2} \boldsymbol{\tau}^{2}\right) .
$$

In the reference configuration

$$
I_{9}=I_{11}=R_{1}, \quad I_{10}=I_{12}=R_{1}^{2}-2 R_{2} .
$$


Altogether there are four second-order tensors involved here, namely $\mathbf{C}, \mathbf{M} \otimes \mathbf{M}, \mathbf{M}^{\prime} \otimes \mathbf{M}^{\prime}$ and $\boldsymbol{\tau}$, and the associated full integrity basis requires a set of independent invariants of combinations of two, three and all four tensors in addition to those of $\mathbf{C}$ and $\boldsymbol{\tau}$ listed above. The invariants that are independent of $\mathbf{M}^{\prime}$ are

$$
\mathbf{M} \cdot(\mathbf{C} \boldsymbol{\tau} \mathbf{M}), \quad \mathbf{M} \cdot\left(\mathbf{C} \boldsymbol{\tau}^{2} \mathbf{M}\right), \quad \mathbf{M} \cdot\left(\mathbf{C}^{2} \boldsymbol{\tau} \mathbf{M}\right), \quad \mathbf{M} \cdot\left(\mathbf{C}^{2} \boldsymbol{\tau}^{2} \mathbf{M}\right)
$$

while those which are independent of $\mathbf{M}$ are

$$
\mathbf{M}^{\prime} \cdot\left(\mathbf{C} \boldsymbol{\tau} \mathbf{M}^{\prime}\right), \quad \mathbf{M}^{\prime} \cdot\left(\mathbf{C} \tau^{2} \mathbf{M}^{\prime}\right), \quad \mathbf{M}^{\prime} \cdot\left(\mathbf{C}^{2} \boldsymbol{\tau} \mathbf{M}^{\prime}\right), \quad \mathbf{M}^{\prime} \cdot\left(\mathbf{C}^{2} \boldsymbol{\tau}^{2} \mathbf{M}^{\prime}\right) .
$$

These reduce to $R_{4}, R_{5}, R_{6}$ or $R_{7}$, as appropriate, in the reference configuration

Invariants that depend on all four tensors include, apart from a factor $\mathbf{M} \cdot \mathbf{M}^{\prime}$

$$
\mathbf{M} \cdot\left(\mathbf{C} \tau \mathbf{M}^{\prime}\right), \quad \mathbf{M} \cdot\left(\tau \mathbf{C M}^{\prime}\right), \quad \mathbf{M} \cdot\left(\mathbf{C}^{2} \boldsymbol{\tau} \mathbf{M}^{\prime}\right), \quad \mathbf{M} \cdot\left(\boldsymbol{\tau}^{2} \mathbf{C M}^{\prime}\right)
$$

and

$$
\mathbf{M} \cdot\left(\mathbf{C}^{2} \boldsymbol{\tau}^{2} \mathbf{M}^{\prime}\right), \quad \mathbf{M} \cdot\left(\boldsymbol{\tau}^{2} \mathbf{C}^{2} \mathbf{M}^{\prime}\right) .
$$

Terms including $\mathbf{M} \cdot\left(\mathbf{C} \boldsymbol{\tau} \mathbf{C M}^{\prime}\right), \mathbf{M} \cdot\left(\mathbf{C} \boldsymbol{\tau}^{2} \mathbf{C M}^{\prime}\right), \mathbf{M} \cdot\left(\mathbf{C}^{2} \boldsymbol{\tau} \mathbf{C}^{2} \mathbf{M}^{\prime}\right)$ and $\mathbf{M} \cdot\left(\mathbf{C} \boldsymbol{\tau} \mathbf{C} \boldsymbol{\tau} \mathbf{M}^{\prime}\right)$, for example, and those obtained by reversing the roles of $\boldsymbol{\tau}$ and $\mathbf{C}$ can be expressed in terms of the previously listed invariants, as exemplified in Appendix A, in which a procedure has been outlined for obtaining connections between the invariants. However, illustration of the theory would be far too complicated if all such invariants were to be included, and for the application considered in the following sections a considerably reduced set of invariants will be adopted, but a set that accounts for both preferred directions and the residual stress.

\subsection{Hyperelastic materials}

The theory of hyperelasticity describes the elastic response of a material using a strain-energy function, defined per unit volume in the reference configuration $\mathcal{B}_{\mathrm{r}}$, which we denote by $W$. In the absence of residual stress and preferred directions, for a homogeneous material $W$ depends only on the deformation gradient $\mathbf{F}$, and, by objectivity, it depends of $\mathbf{F}$ through $\mathbf{C}$, and we write $W=W(\mathbf{C})$. For a residually stressed, anisotropic elastic solid with two preferred directions, $W$ depends on $\mathbf{C}$, on the structure tensors $\mathbf{M} \otimes \mathbf{M}$, 
$\mathbf{M}^{\prime} \otimes \mathbf{M}^{\prime}$ and also on the residual stress $\boldsymbol{\tau}$, and we express this dependence by writing

$$
W=W\left(\mathbf{C}, \mathbf{M} \otimes \mathbf{M}, \mathbf{M}^{\prime} \otimes \mathbf{M}^{\prime}, \boldsymbol{\tau}\right) .
$$

The nominal and Cauchy stress tensors, denoted $\mathbf{S}$ and $\boldsymbol{\sigma}$ respectively, are given by the standard formulas

$$
\mathbf{S}=\frac{\partial W}{\partial \mathbf{F}}, \quad \boldsymbol{\sigma}=J^{-1} \mathbf{F} \frac{\partial W}{\partial \mathbf{F}},
$$

$\boldsymbol{\sigma}$ being symmetric, or in Cartesian components

$$
S_{\alpha i}=\frac{\partial W}{\partial F_{i \alpha}}, \quad \sigma_{i j}=\sigma_{i j}=J^{-1} F_{i \alpha} \frac{\partial W}{\partial F_{j \alpha}},
$$

the first of which identifies the convention used here for the order of the indices in the differentiation with respect to a non-symmetric second-order tensor. Here and henceforth, the summation convention for repeated indices is used.

In the reference configuration the stress calculated from (18) and (19) must recover the residual stress in that configuration, so that, with the arguments included, we must have

$$
\boldsymbol{\tau}=\frac{\partial W}{\partial \mathbf{F}}\left(\mathbf{I}_{\mathrm{r}}, \mathbf{M} \otimes \mathbf{M}, \mathbf{M}^{\prime} \otimes \mathbf{M}^{\prime}, \boldsymbol{\tau}\right),
$$

where $\mathbf{I}_{\mathrm{r}}$ is the identity tensor in $\mathcal{B}_{\mathrm{r}}$. We shall return to this restriction later.

It is sometimes convenient to separate the dependence of $W$ on $\mathbf{F}$ into dilatational and volume preserving contributions, and for this purpose, following Flory (1961) and Ogden (1978), we introduce the multiplicative decomposition

$$
\mathbf{F}=J^{1 / 3} \overline{\mathbf{F}}
$$

where $\overline{\mathbf{F}}$ is the isochoric contribution to the deformation, satisfying $\operatorname{det} \overline{\mathbf{F}}=1$, and $J$ describes the dilatation. For further discussion of this decomposition we refer to Ogden (1997) and Holzapfel (2000). The isochoric right and left Cauchy-Green tensors are, respectively, given by

$$
\overline{\mathbf{C}}=\overline{\mathbf{F}}^{\mathrm{T}} \overline{\mathbf{F}}, \quad \overline{\mathbf{B}}=\overline{\mathbf{F}} \overline{\mathbf{F}}^{\mathrm{T}} .
$$

To account for the decomposition in the material properties we separate the dependence on $\mathbf{C}$ into dependence on $\overline{\mathbf{C}}$ and $J$, and define the modified strain-energy function $\bar{W}$ by

$$
\bar{W}\left(\overline{\mathbf{C}}, J, \mathbf{M} \otimes \mathbf{M}, \mathbf{M}^{\prime} \otimes \mathbf{M}^{\prime}, \boldsymbol{\tau}\right)=W\left(\mathbf{C}, \mathbf{M} \otimes \mathbf{M}, \mathbf{M}^{\prime} \otimes \mathbf{M}^{\prime}, \boldsymbol{\tau}\right),
$$


from which, following Ogden (1978), the nominal and Cauchy stresses can be calculated as

$$
\begin{gathered}
\mathbf{S}=J^{-1 / 3}\left[\frac{\partial \bar{W}}{\partial \overline{\mathbf{F}}}-\frac{1}{3} \operatorname{tr}\left(\overline{\mathbf{F}} \frac{\partial \bar{W}}{\partial \overline{\mathbf{F}}}\right) \overline{\mathbf{F}}^{-1}\right]+J^{2 / 3} \frac{\partial \bar{W}}{\partial J} \overline{\mathbf{F}}^{-1}, \\
\boldsymbol{\sigma}=J^{-1}\left[\overline{\mathbf{F}} \frac{\partial \bar{W}}{\partial \overline{\mathbf{F}}}-\frac{1}{3} \operatorname{tr}\left(\overline{\mathbf{F}} \frac{\partial \bar{W}}{\partial \overline{\mathbf{F}}}\right) \mathbf{I}\right]+\frac{\partial \bar{W}}{\partial J} \mathbf{I}
\end{gathered}
$$

where $\mathbf{I}$ is the identity tensor in $\mathcal{B}$. From the latter it follows that

$$
\frac{\partial \bar{W}}{\partial J}=\frac{1}{3} \operatorname{tr} \boldsymbol{\sigma}
$$

the hydrostatic part of the stress, a result given in Ogden (1978) and for an isotropic material in Penn (1970).

For the considered structure the strain energy must be an isotropic function of the four tensors $\mathbf{C}, \mathbf{M} \otimes \mathbf{M}, \mathbf{M}^{\prime} \otimes \mathbf{M}^{\prime}, \boldsymbol{\tau}$, and $W$ is then reduced to dependence on the invariants of the tensors listed in Sections 2.1-2.3. Equivalently, it can be regarded as depending on the isochoric invariants together with $J$. We distinguish the isochoric invariants with an overbar. Thus, by replacing $\mathbf{C}$ by $\overline{\mathbf{C}}$ in the definitions of the invariants involving $\mathbf{C}$ we obtain $\bar{I}_{3}=1$ and

$$
\bar{I}_{i}= \begin{cases}J^{-2 / 3} I_{i}, & i=1,4,6,9,10, \\ J^{-4 / 3} I_{i}, & i=2,5,7,11,12,\end{cases}
$$

and similarly for the other invariants, for which we have not adopted particular notations.

For a simple illustration that includes dependence on both preferred directions and the residual stress, we consider an energy function that depends only on the invariants $\bar{I}_{1}, \bar{I}_{4}, \bar{I}_{6}$ and $\bar{I}_{9}$, along with $J$ and the collection $R$ (or a subset of $R$ ) of invariants independent of $\mathbf{C}$ :

$$
W\left(I_{1}, I_{3}, I_{4}, I_{6}, I_{9}, R\right)=\bar{W}\left(\bar{I}_{1}, \bar{I}_{4}, \bar{I}_{6}, \bar{I}_{9}, J, R\right) .
$$

The expression for the Cauchy stress based on $W$ may be expanded as

$$
\boldsymbol{\sigma}=2 J^{-1}\left[W_{1} \mathbf{B}+I_{3} W_{3} \mathbf{I}+W_{4} \mathbf{m} \otimes \mathbf{m}+W_{6} \mathbf{m}^{\prime} \otimes \mathbf{m}^{\prime}+W_{9} \boldsymbol{\Sigma}\right],
$$

where $\mathbf{m}=\mathbf{F M}, \mathbf{m}^{\prime}=\mathbf{F M}^{\prime}, \boldsymbol{\Sigma}=\mathbf{F} \boldsymbol{\tau} \mathbf{F}^{\mathrm{T}}$, which are, respectively, the push forward versions of $\mathbf{M}, \mathbf{M}^{\prime}$ and $\boldsymbol{\tau}$ from $\mathcal{B}_{\mathrm{r}}$ to $\mathcal{B}$. Here $W_{i}=\partial W / \partial I_{i}, i=$ $1,3,4,6,9$. 
Alternatively, based on $\bar{W}$, and with $\bar{W}_{i}=\partial \bar{W} / \partial \bar{I}_{i}, i=1,4,6,9$, and $\bar{W}_{J}=\partial \bar{W} / \partial J$

$$
\begin{aligned}
\boldsymbol{\sigma}=2 J^{-1}\left[\bar{W}_{1} \operatorname{dev} \overline{\mathbf{B}}\right. & +\bar{W}_{4} \operatorname{dev}(\overline{\mathbf{m}} \otimes \overline{\mathbf{m}}) \\
& \left.+\bar{W}_{6} \operatorname{dev}\left(\overline{\mathbf{m}}^{\prime} \otimes \overline{\mathbf{m}}^{\prime}\right)+\bar{W}_{9} \operatorname{dev} \overline{\mathbf{\Sigma}}\right]+\bar{W}_{J} \mathbf{I},
\end{aligned}
$$

in which we have made use of the formulas

$$
\begin{gathered}
\mathbf{F} \frac{\partial \bar{I}_{1}}{\partial \mathbf{F}}=2 \operatorname{dev} \overline{\mathbf{B}}, \quad \mathbf{F} \frac{\partial \bar{I}_{4}}{\partial \mathbf{F}}=2 \operatorname{dev}(\overline{\mathbf{m}} \otimes \overline{\mathbf{m}}), \\
\mathbf{F} \frac{\partial \bar{I}_{6}}{\partial \mathbf{F}}=2 \operatorname{dev}\left(\overline{\mathbf{m}}^{\prime} \otimes \overline{\mathbf{m}}^{\prime}\right), \quad \mathbf{F} \frac{\partial \bar{I}_{9}}{\partial \mathbf{F}}=2 \operatorname{dev} \overline{\boldsymbol{\Sigma}},
\end{gathered}
$$

where dev indicates the deviatoric part of a second-order tensor defined by

$$
\operatorname{dev}(\bullet)=(\bullet)-\frac{1}{3} \operatorname{tr}(\bullet) \mathbf{I}
$$

and $\overline{\mathbf{m}}=\overline{\mathbf{F}} \mathbf{M}, \overline{\mathbf{m}}^{\prime}=\overline{\mathbf{F}} \mathbf{M}^{\prime}, \bar{\Sigma}=\overline{\mathbf{F}} \boldsymbol{\tau} \overline{\mathbf{F}}^{\mathrm{T}}$.

In the reference configuration the specialization of (21) becomes, from (30),

$$
\boldsymbol{\tau}=2\left(W_{1}+W_{3}\right) \mathbf{I}_{\mathrm{r}}+2 W_{4} \mathbf{M} \otimes \mathbf{M}+2 W_{6} \mathbf{M}^{\prime} \otimes \mathbf{M}^{\prime}+2 W_{9} \boldsymbol{\tau}
$$

while from (31), noting that $\operatorname{dev} \overline{\mathbf{B}}=\mathbf{0}$ in the reference configuration,

$$
\boldsymbol{\tau}=2 \bar{W}_{4} \operatorname{dev}(\mathbf{M} \otimes \mathbf{M})+2 \bar{W}_{6} \operatorname{dev}\left(\mathbf{M}^{\prime} \otimes \mathbf{M}^{\prime}\right)+2 \bar{W}_{9} \operatorname{dev} \boldsymbol{\tau}+\bar{W}_{J} \mathbf{I}_{\mathrm{r}}
$$

all the derivatives being evaluated in the reference configuration, where $I_{1}=$ $3, I_{3}=1, I_{4}=I_{6}=1, I_{9}=\operatorname{tr} \boldsymbol{\tau}$.

It may be deduced from (35) that

$$
W_{1}+W_{3}=0, \quad W_{4}=W_{6}=0, \quad 2 W_{9}=1,
$$

in the reference configuration, and hence $\bar{W}_{4}=\bar{W}_{6}=0,2 \bar{W}_{9}=1$. In the absence of preferred directions the conditions (37) are special cases of those given in Shams et al. (2011). It follows from (36) that in the reference configuration the condition

$$
\bar{W}_{J}=\frac{1}{3} \operatorname{tr} \tau
$$

must be satisfied. 
Following a common practice in computational mechanics, we decouple the energy function into the sum of a part that depends on $\overline{\mathbf{C}}$ and a part that depends on $J$. Here we express this in the form

$$
\bar{W}=\bar{W}_{\text {iso }}\left(\overline{\mathbf{C}}, \mathbf{M} \otimes \mathbf{M}, \mathbf{M}^{\prime} \otimes \mathbf{M}^{\prime}, \boldsymbol{\tau}\right)+\bar{W}_{\text {vol }}(J, R),
$$

where $\bar{W}_{\text {iso }}$ and $\bar{W}_{\text {vol }}$ denote the volume-preserving (isochoric) and volumetric (dilatational) contributions. Note that in general we allow $\bar{W}_{\text {vol }}$ to depend of the set of invariants $R$ as well as $J$.

We now specialize this to the particular model involving $\bar{I}_{1}, \bar{I}_{4}, \bar{I}_{6}, \bar{I}_{9}, J$ that we considered earlier, so that

$$
\bar{W}=\bar{W}_{\text {iso }}\left(\bar{I}_{1}, \bar{I}_{4}, \bar{I}_{6}, \bar{I}_{9}, R\right)+\bar{W}_{\text {vol }}(J, R) .
$$

From (31) we are then able to decompose the Cauchy stress into the sum of an isochoric part (apart from the factor $J^{-1}$ ), denoted $\overline{\boldsymbol{\sigma}}$, and a volumetric part, denoted $\boldsymbol{\sigma}_{\mathrm{vol}}$, so that $\boldsymbol{\sigma}=\overline{\boldsymbol{\sigma}}+\boldsymbol{\sigma}_{\mathrm{vol}}$, where

$$
\overline{\boldsymbol{\sigma}}=2 J^{-1}\left[\bar{W}_{1} \operatorname{dev} \overline{\mathbf{B}}+\bar{W}_{4} \operatorname{dev}(\overline{\mathbf{m}} \otimes \overline{\mathbf{m}})+\bar{W}_{6} \operatorname{dev}\left(\overline{\mathbf{m}}^{\prime} \otimes \overline{\mathbf{m}}^{\prime}\right)+\bar{W}_{9} \operatorname{dev} \overline{\boldsymbol{\Sigma}}\right]
$$

and

$$
\boldsymbol{\sigma}_{\mathrm{vol}}=\bar{W}_{J} \mathbf{I}
$$

\section{Towards numerical implementation}

Exact solutions of boundary-value problems for materials subject to residual stress are possible only for a limited number with simple geometries, constitutive models and residual stress distributions, as exemplified in Merodio et al. (2013), Merodio and Ogden (2015) and Ogden (2015). In general, therefore, numerical solutions are required, and in this section we develop a tensor framework suitable for implementation of a nearly incompressible, anisotropic and residually stressed material model in the finite element package Abaqus (2013).

For this purpose we consider a small incremental displacement, denoted $\dot{\mathrm{x}}=\dot{\boldsymbol{\chi}}(\mathbf{X})$, superimposed on the configuration $\mathcal{B}$, where here and in the following a superposed dot signifies an incremental quantity. By using the inverse deformation $\mathbf{X}=\chi^{-1}(\mathbf{x}), \dot{\mathbf{x}}$ can be expressed in Eulerian form as a function $\mathbf{u}(\mathbf{x})=\dot{\chi}\left(\chi^{-1}(\mathbf{x})\right)$. This leads to the important connection

$$
\dot{\mathbf{F}}=\mathbf{L F}, \quad \mathbf{L}=\operatorname{grad} \mathbf{u}
$$


for the increment of the deformation gradient.

The tensor $\mathbf{L}$, the spatial gradient of $\mathbf{u}$, has Cartesian components $L_{i j}=$ $\partial u_{i} / \partial x_{j}$. Its symmetric and antisymmetric parts are denoted $\mathbf{D}$ and $\mathbf{W}$, respectively, and given by

$$
\mathbf{D}=\frac{1}{2}\left(\mathbf{L}+\mathbf{L}^{\mathrm{T}}\right), \quad \mathbf{W}=\frac{1}{2}\left(\mathbf{L}-\mathbf{L}^{\mathrm{T}}\right) .
$$

Abaqus makes use of the Jaumann rate of the Kirchhoff stress $J \boldsymbol{\sigma}$ and the associated elasticity tensor. Here we consider the Jaumann increment, denoted $\mathcal{D}(J \boldsymbol{\sigma})$, of the Kirchhoff stress. This is related to the increment $\frac{\dot{J} \boldsymbol{\sigma}}{}$ by

$$
\mathcal{D}(J \boldsymbol{\sigma})=\dot{\bar{J} \boldsymbol{\sigma}}-J(\mathbf{W} \boldsymbol{\sigma}-\boldsymbol{\sigma} \mathbf{W}),
$$

and the associated (fourth-order) elasticity tensor, denoted $\mathbb{C}$, comes from the connection

$$
\mathcal{D}(J \boldsymbol{\sigma})=\mathbb{C}: \mathbf{D}
$$

where the operator : represents the standard double contraction, defined in index notation by $\mathbf{A}: \mathbf{B}=\operatorname{tr}\left(\mathbf{A B}^{\mathrm{T}}\right)=A_{i j} B_{i j}$ for two second-order tensors, or $(\mathbb{C}: \mathbf{D})_{i j}=\mathbb{C}_{i j k l} D_{k l}$ in the above. Since the contractions considered in the following involve only symmetric tensors the transpose can be omitted.

Thus, we have, from equations (45) and (46),

$$
\dot{\bar{J} \boldsymbol{\sigma}}=\mathbb{C}: \mathbf{D}+J(\mathbf{W} \boldsymbol{\sigma}-\boldsymbol{\sigma} \mathbf{W}) .
$$

For the decoupled model (39), on which we focus from now on, the stress increment $\dot{\bar{J} \boldsymbol{\sigma}}$ can be decomposed into isochoric and volumetric components, which are denoted respectively by $\dot{\bar{J} \overline{\boldsymbol{\sigma}}}$ and $\dot{\overline{J \boldsymbol{\sigma}}}_{\mathrm{vol}}$. Hence, from equation (47) it follows that

$$
\dot{\bar{J} \overline{\boldsymbol{\sigma}}}=\overline{\mathbb{C}}: \mathbf{D}+J(\mathbf{W} \overline{\boldsymbol{\sigma}}-\overline{\boldsymbol{\sigma}} \mathbf{W}),
$$

and

$$
\dot{\bar{J}}_{\mathrm{vol}}=\mathbb{C}_{\mathrm{vol}}: \mathbf{D}
$$

where $\overline{\mathbb{C}}$ is the isochoric and $\mathbb{C}_{\mathrm{vol}}$ the volumetric part of the elasticity tensor, i.e. $\mathbb{C}=\overline{\mathbb{C}}+\mathbb{C}_{\text {vol }}$. We now calculate $\dot{\bar{J} \overline{\boldsymbol{\sigma}}}$ and $\frac{\dot{J}}{\bar{J} \boldsymbol{\sigma}_{\text {vol }}}$ directly from (41) and (42) in order to obtain explicit expressions for $\overline{\mathbb{C}}$ and $\mathbb{C}_{\text {vol }}$. 


\subsection{An elasticity tensor}

With attention restricted to the decoupled energy function (39) and the Cauchy stress given by (31), the increment of the isochoric part (41) can be written as

$$
\begin{aligned}
\dot{J \overline{\boldsymbol{\sigma}}} & =2\left[\dot{\bar{W}}_{1} \operatorname{dev} \overline{\mathbf{B}}+\bar{W}_{1} \frac{\cdot}{\operatorname{dev} \overline{\mathbf{B}}}+\dot{\bar{W}}_{4} \operatorname{dev}(\overline{\mathbf{m}} \otimes \overline{\mathbf{m}})+\bar{W}_{4} \overline{\operatorname{dev}(\overline{\mathbf{m}} \otimes \overline{\mathbf{m}})}\right. \\
& \left.+\dot{\bar{W}}_{6} \operatorname{dev}\left(\overline{\mathbf{m}}^{\prime} \otimes \overline{\mathbf{m}}^{\prime}\right)+\bar{W}_{6} \frac{.}{\operatorname{dev}\left(\overline{\mathbf{m}}^{\prime} \otimes \overline{\mathbf{m}}^{\prime}\right)}+\dot{\bar{W}}_{9} \operatorname{dev} \overline{\boldsymbol{\Sigma}}+\bar{W}_{9} \frac{.}{\operatorname{dev} \overline{\boldsymbol{\Sigma}}}\right] .
\end{aligned}
$$

For convenience, the expressions $\dot{\bar{W}}_{i}, i \in\{1,4,6,9\}$, are written as

$$
\dot{\bar{W}}_{i}=\sum_{j=1,4,6,9} \bar{W}_{i j} \dot{\bar{I}}_{j}, \quad i \in\{1,4,6,9\}
$$

where $\bar{W}_{i j}=\partial^{2} \bar{W} / \partial \bar{I}_{i} \partial \bar{I}_{j}, i, j \in\{1,4,6,9\}$. The increments of the invariants are given by

$$
\begin{aligned}
& \dot{\bar{I}}_{1}=2 \operatorname{dev} \overline{\mathbf{B}}: \mathbf{D}, \quad \dot{\bar{I}}_{4}=2 \operatorname{dev}(\overline{\mathbf{m}} \otimes \overline{\mathbf{m}}): \mathbf{D} \\
& \dot{\bar{I}}_{6}=2 \operatorname{dev}\left(\overline{\mathbf{m}}^{\prime} \otimes \overline{\mathbf{m}}^{\prime}\right): \mathbf{D}, \quad \dot{\bar{I}}_{9}=2 \operatorname{dev} \overline{\mathbf{\Sigma}}: \mathbf{D}
\end{aligned}
$$

From the definition of $\operatorname{dev} \overline{\mathbf{B}}$ it follows that its increment is given by

$$
\overline{\operatorname{dev} \overline{\mathbf{B}}}=\operatorname{dev} \dot{\overline{\mathbf{B}}}
$$

while the increment of $\overline{\mathbf{B}}$ can be written as

$$
\dot{\overline{\mathbf{B}}}=\mathbb{H}: \operatorname{dev} \mathbf{D}+\mathbf{W} \overline{\mathbf{B}}-\overline{\mathbf{B}} \mathbf{W},
$$

where the fourth-order tensor $\mathbb{H}$ has the component form

$$
\mathbb{H}_{i j k l}=\frac{1}{2}\left(\delta_{i k} \bar{B}_{j l}+\delta_{i l} \bar{B}_{j k}+\bar{B}_{i k} \delta_{j l}+\bar{B}_{i l} \delta_{j k}\right) .
$$

Equation (53) can now be written

$$
\overline{\operatorname{dev} \overline{\mathbf{B}}}=\operatorname{dev}(\mathbb{H}: \operatorname{dev} \mathbf{D})+\mathbf{W} \overline{\mathbf{B}}-\overline{\mathbf{B}} \mathbf{W},
$$

and hence

$$
\overline{\operatorname{dev} \overline{\mathbf{B}}}=\mathbb{B}: \mathbf{D}+\mathbf{W} \overline{\mathbf{B}}-\overline{\mathbf{B}} \mathbf{W}
$$


where, for convenience, we have introduced the fourth-order tensor $\mathbb{B}$ defined by

$$
\mathbb{B}=\mathbb{H}-\frac{2}{3}(\mathbf{I} \otimes \overline{\mathbf{B}}+\overline{\mathbf{B}} \otimes \mathbf{I})+\frac{2}{9} \bar{I}_{1} \mathbf{I} \otimes \mathbf{I} .
$$

Following a similar process to that leading to equation (57), the increments of $\operatorname{dev}(\overline{\mathbf{m}} \otimes \overline{\mathbf{m}}), \operatorname{dev}\left(\overline{\mathbf{m}}^{\prime} \otimes \overline{\mathbf{m}}^{\prime}\right)$ and $\operatorname{dev} \overline{\mathbf{\Sigma}}$ can be written as

$$
\begin{aligned}
\overline{\operatorname{dev}(\overline{\mathbf{m}} \otimes \overline{\mathbf{m}})} & =\mathbf{M}: \mathbf{D}+\mathbf{W}(\overline{\mathbf{m}} \otimes \overline{\mathbf{m}})-(\overline{\mathbf{m}} \otimes \overline{\mathbf{m}}) \mathbf{W}, \\
\frac{\operatorname{dev}\left(\overline{\mathbf{m}}^{\prime} \otimes \overline{\mathbf{m}}^{\prime}\right)}{\frac{\cdot}{\operatorname{dev} \bar{\Sigma}}} & =\mathbf{M}^{\prime}: \mathbf{D}+\mathbf{W}\left(\overline{\mathbf{m}}^{\prime} \otimes \overline{\mathbf{m}}^{\prime}\right)-\left(\overline{\mathbf{m}}^{\prime} \otimes \overline{\mathbf{m}}^{\prime}\right) \mathbf{W}, \mathbf{W} \overline{\mathbf{\Sigma}}-\overline{\mathbf{\Sigma}} \mathbf{W},
\end{aligned}
$$

where $\mathbf{M}, \mathbf{M}^{\prime}$ and $\mathbf{S}$, respectively, are given in component form by

$$
\begin{aligned}
\mathbb{M}_{i j k l} & =\frac{1}{2}\left(\delta_{i k} \bar{m}_{j} \bar{m}_{l}+\bar{m}_{i} \bar{m}_{k} \delta_{j l}+\delta_{i l} \bar{m}_{j} \bar{m}_{k}+\bar{m}_{i} \bar{m}_{l} \delta_{j k}\right) \\
& -\frac{2}{3}\left(\delta_{i j} \bar{m}_{k} \bar{m}_{l}+\bar{m}_{i} \bar{m}_{j} \delta_{k l}\right)+\frac{2}{9} \bar{I}_{4} \delta_{i j} \delta_{k l}, \\
\mathbb{M}_{i j k l}^{\prime} & =\frac{1}{2}\left(\delta_{i k} \bar{m}_{j}^{\prime} \bar{m}_{l}^{\prime}+\bar{m}_{i}^{\prime} \bar{m}_{k}^{\prime} \delta_{j l}+\delta_{i l} \bar{m}_{j}^{\prime} \bar{m}_{k}^{\prime}+\bar{m}_{i}^{\prime} \bar{m}_{l}^{\prime} \delta_{j k}\right) \\
& -\frac{2}{3}\left(\delta_{i j} \bar{m}_{k}^{\prime} \bar{m}_{l}^{\prime}+\bar{m}_{i}^{\prime} \bar{m}_{j}^{\prime} \delta_{k l}\right)+\frac{2}{9} \bar{I}_{6} \delta_{i j} \delta_{k l}, \\
\mathbb{S}_{i j k l} & =\frac{1}{2}\left(\delta_{i k} \bar{\Sigma}_{j l}+\bar{\Sigma}_{i k} \delta_{j l}+\delta_{i l} \bar{\Sigma}_{j k}+\bar{\Sigma}_{i l} \delta_{j k}\right) \\
& -\frac{2}{3}\left(\delta_{i j} \bar{\Sigma}_{k l}+\bar{\Sigma}_{i j} \delta_{k l}\right)+\frac{2}{9} \bar{I}_{9} \delta_{i j} \delta_{k l} .
\end{aligned}
$$

By first substituting (52) into (51) and then (51) and (57)-(61) into (50) and then comparing the result with equation (48) the explicit expression for the isochoric part $\overline{\mathbb{C}}$ of the elasticity tensor is obtained as

$$
\begin{aligned}
\overline{\mathbf{C}} & =4\left\{\bar{W}_{11} \operatorname{dev} \overline{\mathbf{B}} \otimes \operatorname{dev} \overline{\mathbf{B}}+\bar{W}_{14}[\operatorname{dev} \overline{\mathbf{B}} \otimes \operatorname{dev}(\overline{\mathbf{m}} \otimes \overline{\mathbf{m}})\right. \\
& +\operatorname{dev}(\overline{\mathbf{m}} \otimes \overline{\mathbf{m}}) \otimes \operatorname{dev} \overline{\mathbf{B}}]+\bar{W}_{16}\left[\operatorname{dev} \overline{\mathbf{B}} \otimes \operatorname{dev}\left(\overline{\mathbf{m}}^{\prime} \otimes \overline{\mathbf{m}}^{\prime}\right)\right. \\
& \left.+\operatorname{dev}\left(\overline{\mathbf{m}}^{\prime} \otimes \overline{\mathbf{m}}^{\prime}\right) \otimes \operatorname{dev} \overline{\mathbf{B}}\right]+\bar{W}_{19}[\operatorname{dev} \overline{\mathbf{B}} \otimes \operatorname{dev} \overline{\boldsymbol{\Sigma}}+\operatorname{dev} \overline{\boldsymbol{\Sigma}} \otimes \operatorname{dev} \overline{\mathbf{B}}] \\
& +\bar{W}_{44} \operatorname{dev}(\overline{\mathbf{m}} \otimes \overline{\mathbf{m}}) \otimes \operatorname{dev}(\overline{\mathbf{m}} \otimes \overline{\mathbf{m}})+\bar{W}_{66} \operatorname{dev}\left(\overline{\mathbf{m}}^{\prime} \otimes \overline{\mathbf{m}}^{\prime}\right) \otimes \operatorname{dev}\left(\overline{\mathbf{m}}^{\prime} \otimes \overline{\mathbf{m}}^{\prime}\right) \\
& +\bar{W}_{46}\left[\operatorname{dev}(\overline{\mathbf{m}} \otimes \overline{\mathbf{m}}) \otimes \operatorname{dev}\left(\overline{\mathbf{m}}^{\prime} \otimes \overline{\mathbf{m}}^{\prime}\right)+\operatorname{dev}\left(\overline{\mathbf{m}}^{\prime} \otimes \overline{\mathbf{m}}^{\prime}\right) \otimes \operatorname{dev}(\overline{\mathbf{m}} \otimes \overline{\mathbf{m}})\right] \\
& +\bar{W}_{49}[\operatorname{dev}(\overline{\mathbf{m}} \otimes \overline{\mathbf{m}}) \otimes \operatorname{dev} \overline{\mathbf{\Sigma}}+\operatorname{dev} \overline{\boldsymbol{\Sigma}} \otimes \operatorname{dev}(\overline{\mathbf{m}} \otimes \overline{\mathbf{m}})] \\
& +\bar{W}_{69}\left[\operatorname{dev}\left(\overline{\mathbf{m}}^{\prime} \otimes \overline{\mathbf{m}}^{\prime}\right) \otimes \operatorname{dev} \overline{\mathbf{\Sigma}}+\operatorname{dev} \overline{\boldsymbol{\Sigma}} \otimes \operatorname{dev}\left(\overline{\mathbf{m}}^{\prime} \otimes \overline{\mathbf{m}}^{\prime}\right)\right] \\
& \left.+\bar{W}_{99} \operatorname{dev} \overline{\boldsymbol{\Sigma}} \otimes \operatorname{dev} \overline{\mathbf{\Sigma}}\right\}+2\left(\bar{W}_{1} \mathbb{B}+\bar{W}_{4} \mathbb{M}+\bar{W}_{6} \mathbf{M}^{\prime}+\bar{W}_{9} \mathbf{S}\right),
\end{aligned}
$$

wherein the derivatives of $\bar{W}$ are the same as those of $\bar{W}_{\text {iso }}$.

The increment of the volumetric part of the Cauchy stress $J \boldsymbol{\sigma}_{\mathrm{vol}}$ is given by

$$
\frac{\cdot}{J \boldsymbol{\sigma}_{\mathrm{vol}}}=\dot{J}\left(\frac{\mathrm{d} \bar{W}_{\mathrm{vol}}}{\mathrm{d} J}+J \frac{\mathrm{d}^{2} \bar{W}_{\text {vol }}}{\mathrm{d} J^{2}}\right) \mathbf{I}
$$


where the increment of $J$ is given by $\dot{J}=J \operatorname{tr} \mathbf{D}$. Comparison of this with equation (49) enables $\mathbb{C}_{\mathrm{vol}}$ to be expressed in the form

$$
\mathbb{C}_{\text {vol }}=J\left(\frac{\mathrm{d} \bar{W}_{\text {vol }}}{\mathrm{d} J}+J \frac{\mathrm{d}^{2} \bar{W}_{\text {vol }}}{\mathrm{d} J^{2}}\right) \mathbf{I} \otimes \mathbf{I} .
$$

The total elasticity tensor is then obtained as $\mathbb{C}=\overline{\mathbb{C}}+\mathbb{C}_{\text {vol }}$.

\subsection{Constitutive model}

In order to implement the model within a finite element framework a particular form of the decoupled energy function $\bar{W}$ in (39) is required. For definiteness we choose $\bar{W}_{\text {iso }}$ to consist of a contribution from the isotropic base matrix, and anisotropic contributions from two families of oriented fibres and from the residual stress. We take the response of the isotropic base matrix to be given by the exponential strain-energy function due to Demiray (1972), and the anisotropic character using the formulation developed by Holzapfel et al. (2000). The residual stress is accounted for in a simple way by using a single term, as in Merodio et al. (2013), Ogden (2015) and Merodio and Ogden (2015), following a slightly more general form adopted in Shams et al. (2011).

The isochoric part $\bar{W}_{\text {iso }}$ of the energy function can then be written in the form

$$
\begin{aligned}
\bar{W}_{\text {iso }} & =\frac{\mu}{2 \alpha}\left\{\exp \left[\alpha\left(\bar{I}_{1}-3\right)\right]-1\right\}+\frac{\mu_{\mathrm{f}}}{2 k} \sum_{i=4,6}\left\{\exp \left[k\left(\bar{I}_{i}-1\right)^{2}\right]-1\right\} \\
& +\frac{1}{2}\left(\bar{I}_{9}-\operatorname{tr} \boldsymbol{\tau}\right),
\end{aligned}
$$

where $\mu$ denotes the shear modulus of the matrix in its reference configuration, $\mu_{\mathrm{f}}$ is related to the degree of anisotropy due to the fibres ( $\mu$ and $\mu_{\mathrm{f}}$ have dimensions of stress) and $\alpha$ and $k$ are dimensionless parameters. The final term is chosen for consistency with the restriction $(37)_{3}$, while the term $\operatorname{tr} \boldsymbol{\tau}$ ensures that $\bar{W}_{\text {iso }}=0$ in the reference configuration.

For the volumetric part $\bar{W}_{\text {vol }}$ of the strain energy there are multiple possible choices, but they must be consistent with (38). We take it to have the form

$$
\bar{W}_{\mathrm{vol}}=\frac{1}{2} \kappa(J-1)^{2}+\frac{1}{3}(J-1) \operatorname{tr} \tau .
$$

The first term is standard in finite element implementations, where $\kappa$ is referred to as a penalty parameter (see, for example, Adler et al., 2014), which, 
for nearly incompressible materials, is taken to be sufficiently large so as to minimize changes in volume. The second term is included for consistency with (38), but $J-1$ therein could be replaced by another suitable function that ensures such consistency, one such being $\log J$.

The Cauchy stress obtained from (31) corresponding to the isochoric and volumetric energy functions (66) and (67) is then given by

$$
\begin{aligned}
\boldsymbol{\sigma} & =J^{-1}\left\{\mu \exp \left[\alpha\left(\bar{I}_{1}-3\right)\right] \operatorname{dev} \overline{\mathbf{B}}+2 \mu_{\mathrm{f}}\left(\bar{I}_{4}-1\right) \exp \left[k\left(\bar{I}_{4}-1\right)^{2}\right] \operatorname{dev}(\overline{\mathbf{m}} \otimes \overline{\mathbf{m}})\right. \\
& \left.+2 \mu_{\mathrm{f}}\left(\bar{I}_{6}-1\right) \exp \left[k\left(\bar{I}_{6}-1\right)^{2}\right] \operatorname{dev}\left(\overline{\mathbf{m}}^{\prime} \otimes \overline{\mathbf{m}}^{\prime}\right)+\operatorname{dev} \overline{\boldsymbol{\Sigma}}\right\} \\
& +\left[\kappa(J-1)+\frac{1}{3} \operatorname{tr} \boldsymbol{\tau}\right] \mathbf{I} .
\end{aligned}
$$

Our objective in the following is to estimate the wall stress in an abdominal aortic aneurysm (AAA) based on a patient-specific geometry combined with a non-uniform distribution of wall pressure using the constitutive law outlined above with residual stress included. Details of the considered wall pressure measurements and the geometrical data are given in Dorfmann et al. (2010).

To the best of our knowledge there are no available data that specify the magnitudes and distributions of the residual stresses in human AAAs. Recent studies have used different approaches to estimate residual stresses. Humphrey and Rajagopal (2002) developed a mixture theory for growth and remodeling of soft tissues. The theory is based on constituents that possess different natural configurations, which generate residual stresses in the absence of applied tractions. They reasoned that it is unlikely that each material particle will have the same natural configuration and therefore the opening angle experiment is a compromise of the individual natural configurations (Saini et al., 1995). A similar conclusion was drawn in Labrosse et al. (2013). Balzani et al. (2007) considered an open arterial ring as the natural configuration and used a numerical method to estimate the stress distribution after the intact ring was reconfigured. Residual stresses in the axial direction were not considered. The method was then applied to an atherosclerotic artery. Balzani et al. (2007) state that the numerical simulation illustrates how to incorporate residual stresses and does not represent a realistic simulation of the residual stresses in arterial walls. Alastrué et al. (2007) presented a methodology for including residual stress fields in arteries having patient-specific geometries. Specifically, they assumed a multiplicative decomposition of the deformation gradient. A sensitivity analysis was performed to evaluate the influence of selected model parameters such 
as axial stretch, the opening angle and the internal radius of the natural configuration. Ambrosi et al. (2008) investigated growth and remodeling processes in an axisymmetric thick-walled cylinder to estimate the residual stress field. The Green strain, given in terms of displacement gradient and a growth stretch tensor, was used in a hyperelastic constitutive law to express Piola stress components. The authors acknowledged the limitations of the model such as the use of isotropic material properties and unrealistic pressure boundary conditions. Polzer et al. (2013) assumed isotropic volumetric growth and estimated the residual strain distribution in the load-free configuration of the vascular wall such that stress differences across the wall are minimized (homogeneous stress hypothesis at mean arterial pressure load). The method, when applied to patient-specific AAA, predicts a tenfold reduction of the von Mises stress gradient across the aneurysmal wall. This is not surprising since the homogeneous stress hypothesis is used to estimate the residual strain distribution. An analytical method was used by Pierce et al. (2015) to estimate the residual stretches in the layered structure of a healthy arterial wall based on the geometry considered in Holzapfel and Ogden (2010). The residual stretches were then used to estimate numerically the residual stress in imaged in vivo geometries of patient specific AAAs.

Bearing in mind that the geometry of typical patient specific AAAs is, in general, very irregular and cannot be approximated by a circular cylindrical tube with constant cross section it is nevertheless useful, as a precursor to consideration of the possible AAA residual stress distribution, to examine first the residual stress in a thick-walled circular cylindrical tube, which we do in the following.

\subsection{Consideration of residual stress in a circular cylindrical tube}

A residual stress must satisfy the equilibrium equation (8) and the boundary condition (9). For a circular cylindrical tube their specializations were used by Merodio et al. (2013), Ogden (2015) and Merodio and Ogden (2015) in which the only nonzero components of the residual stress considered were the radial and azimuthal components, denoted respectively by $\tau_{R R}$ and $\tau_{\Theta \Theta}$, where $R, \Theta$ are the cross-sectional polar coordinates (in the reference configuration). In this situation equation (8) reduces to the radial equation

$$
\frac{\mathrm{d}}{\mathrm{d} R}\left(R \tau_{R R}\right)=\tau_{\Theta \Theta}
$$


and the boundary condition (9) to

$$
\tau_{R R}=0 \quad \text { on } \quad R=A, B,
$$

where $A$ and $B$ are the internal and external radii of the tube in the reference configuration $\mathcal{B}_{\mathrm{r}}$.

The specific form of $\tau_{R R}$ used can be written as

$$
\tau_{R R}=\alpha_{\mathrm{r}}(R-A)(R-B),
$$

where $\alpha_{\mathrm{r}}$ has the units of stress $/(\text { length })^{2}$ and is a measure of the magnitude of the residual stress. The expression (71) satisfies the boundary condition (70). It follows from (69) that $\tau_{\Theta \Theta}$ has the form

$$
\tau_{\Theta \Theta}=\alpha_{\mathrm{r}}\left[3 R^{2}-2(A+B) R+A B\right] .
$$

Plots of $\tau_{R R}$ and $\tau_{\Theta \Theta}$ based on equations (71) and (72) are illustrated in Fig. A.1 for $A=10.7 \mathrm{~mm}, B=12.7 \mathrm{~mm}$, values used in Dorfmann et al. (2010), and $\alpha_{\mathrm{r}}=0.9 \mathrm{kPa} / \mathrm{mm}^{2}$, the latter chosen to approximate stress magnitudes reported in Ogden (2003); Alford et al. (2008); Holzapfel and Ogden (2010).

These residual stress distributions are very similar to those obtained from the opening angle method, as exemplified in Ogden (2003), and are therefore realistic, given that the method is two dimensional and does not consider longitudinal residual stresses.

\section{Patient based AAA model}

\subsection{Material parameters}

In order to determine patient specific values of the material parameters in the isochoric part (66) of the energy function strain-controlled, planar biaxial tests on tissue samples from a AAA resected during elective repair surgery (Pancheri, 2014) were performed. The biaxial specimens are described with reference to cylindrical polar coordinates $(R, \Theta, Z)$, which relate to the local placement of the specimens in a circular cylindrical tube from which they were extracted. The corresponding unit basis vectors $\mathbf{E}_{R}, \mathbf{E}_{\Theta}, \mathbf{E}_{Z}$ are associated with the local radial, azimuthal and axial directions in the tube in its reference (unloaded) configuration. Each specimen is assumed to include two families of parallel fibres oriented at angles $\pm \varphi$ relative to the circumferential 
direction, the unit vector directions being denoted by $\tilde{\mathbf{M}}$ and $\tilde{\mathbf{M}}^{\prime}$ and given by

$$
\tilde{\mathbf{M}}=\cos \varphi \mathbf{E}_{\Theta}+\sin \varphi \mathbf{E}_{Z}, \quad \tilde{\mathbf{M}}^{\prime}=\cos \varphi \mathbf{E}_{\Theta}-\sin \varphi \mathbf{E}_{Z} .
$$

During each test the axial stretch $\lambda_{z}$, corresponding to the direction $\mathbf{E}_{Z}$, was held constant at physiologically realistic values while the specimen was subjected to five loading-unloading cycles in the circumferential direction $\mathbf{E}_{\Theta}$. The nonlinear iterative function fmincon of the commercial software package MATLAB (2014) was used to determine the (non-negative) values of $\mu, \alpha, \mu_{\mathrm{f}}, k, \varphi$ required in (66).

The penalty parameter in the function (67) was taken as $\kappa=20,000 \mathrm{kPa}$. The values of all the material parameters are listed in Table A.1.

\subsection{Computational model of a $A A A$}

A computational model of a patient-based AAA with a non-dilated inner diameter of $21.4 \mathrm{~mm}$, maximum outer diameter of $56.5 \mathrm{~mm}$ and bulge length of $67 \mathrm{~mm}$ was used for the numerical implementation and to analyze the effect of the residual stress on the wall stress distribution at a typical physiological pressure. The wall thickness of the model is assumed to be uniform and set at $2 \mathrm{~mm}$ and the outer diameter of the model entrance and exit to $25.4 \mathrm{~mm}$. Zero displacement boundary conditions were applied at the entrance and exit.

The geometry and finite element structure of the AAA model are shown in Fig. A.2. This is the same structure as in Dorfmann et al. (2010) and uses 63, 654 hexahedral elements connected at 127,926 nodes, with four elements through the wall thickness. We refer to Dorfmann et al. (2010) for a more detailed description.

The pressure distribution applied to the inner surface was obtained from measurements in a patient-based phantom cast at a series of physiologically relevant steady flow rates (Dorfmann et al., 2010). The data show that the wall pressure is $16.0 \mathrm{kPa}$ at the phantom entrance $Z=0$, where $Z$ is the axial position in a global Cartesian coordinate system. At mid-bulge the pressure drops to its minimum of $15.5 \mathrm{kPa}(Z=120-140 \mathrm{~mm})$ and recovers to $15.9 \mathrm{kPa}$ at the bulge exit $(Z=215 \mathrm{~mm})$. The wall pressure applied to the inner surface of the computational model is uniform in the circumferential direction and varies longitudinally. 


\subsection{Fibre orientations in the AAA model}

The unit vectors $\tilde{\mathbf{M}}$ and $\tilde{\mathbf{M}}^{\prime}$ specify the preferred directions in a biaxial specimen, or equivalently locally in an unloaded circular cylindrical tube.

To define the 'corresponding' orientations in the AAA model, we use the projection tensor $\mathcal{P}$, defined by

$$
\mathcal{P}=\mathbf{I}-\mathbf{N} \otimes \mathbf{N},
$$

to generate images of $\tilde{\mathbf{M}}$ and $\tilde{\mathbf{M}}^{\prime}$ projected onto the wall surface defined by the local normal unit vector $\mathbf{N}$. This is accomplished by first identifying a three-dimensional piecewise linear centreline of the AAA. The straight line connecting the centroid of each element to the nearest linear section of the centreline defines the local direction $\mathbf{E}_{R}$, the orientation of the centreline specifies $\mathbf{E}_{Z}$ and their cross product provides $\mathbf{E}_{\Theta}$ based on a small circular cylinder centred locally on the centreline, as depicted in Fig. A.3. Note that $Z$ here is a local cylindrical polar coordinate which should be distinguished from the global Cartesian $Z$. The local orientation of the preferred directions in the AAA model are then given by the unit vectors $\mathbf{M}$ and $\mathbf{M}^{\prime}$ defined by

$$
\mathbf{M}=\frac{\mathcal{P} \tilde{M}}{|\mathcal{P} \tilde{M}|}, \quad \mathbf{M}^{\prime}=\frac{\mathcal{P} \tilde{\mathbf{M}}^{\prime}}{\left|\mathcal{P} \tilde{M}^{\prime}\right|}
$$

It is from these directions that the invariants $\bar{I}_{4}$ and $\bar{I}_{6}$ in the isochoric energy function (66) appropriate for the AAA are calculated.

\subsection{Inclusion of residual stress}

In order to include the residual stress in the AAA we first consider the components $\tau_{R R}$ and $\tau_{\Theta \Theta}$ in a cylinder whose wall thickness is the same as that of the AAA, remembering that in a circular cylinder $\tau_{Z Z}=0$. Then, the residual stress $\boldsymbol{\tau}$ can be written in the spectral form

$$
\boldsymbol{\tau}=\tau_{R R} \mathbf{E}_{R} \otimes \mathbf{E}_{R}+\tau_{\Theta \Theta} \mathbf{E}_{\Theta} \otimes \mathbf{E}_{\Theta},
$$

where $\mathbf{E}_{R}, \mathbf{E}_{\Theta}, \mathbf{E}_{Z}$ are the cylindrical polar axes in the unloaded residually stressed cylinder, as identified in Fig. A.3.

These are mapped onto corresponding unit vectors in the AAA, denoted $\mathbf{E}_{R}^{\prime}=\mathbf{N}, \mathbf{E}_{\Theta}^{\prime}, \mathbf{E}_{Z}^{\prime}$, by means of a rotation tensor, say $\mathbf{R}$, so that

$$
\mathbf{E}_{R}^{\prime}=\mathbf{R E}_{R}, \quad \mathbf{E}_{\Theta}^{\prime}=\mathbf{R E}_{\Theta}, \quad \mathbf{E}_{Z}^{\prime}=\mathbf{R E}_{Z},
$$


$\mathbf{N}$ again being the unit outward normal to the AAA surface.

We map $\boldsymbol{\tau}$ similarly to the AAA as $\mathbf{R} \boldsymbol{\tau} \mathbf{R}^{\mathrm{T}}$, so that

$$
\mathbf{R} \tau \mathbf{R}^{\mathrm{T}}=\tau_{R R} \mathbf{N} \otimes \mathbf{N}+\tau_{\Theta \Theta} \mathbf{E}_{\Theta}^{\prime} \otimes \mathbf{E}_{\Theta}^{\prime}
$$

Hence, the radial component of residual stress becomes the normal component in the AAA and the azimuthal component is locally tangential within the AAA.

It remains to define a suitable $\mathbf{E}_{\Theta}^{\prime}$. Noting that the azimuthal direction in the cylinder is $\mathbf{E}_{\Theta}$, its projection to the $\mathrm{AAA}$ is $\mathcal{P} \mathbf{E}_{\Theta}$. It is therefore appropriate to define $\mathbf{E}_{\Theta}^{\prime}$ as the corresponding unit vector:

$$
\mathbf{E}_{\Theta}^{\prime}=\frac{\mathcal{P} \mathbf{E}_{\Theta}}{\left|\mathcal{P} \mathbf{E}_{\Theta}\right|}
$$

Then $\mathbf{E}_{Z}^{\prime}$ is the unit vector in the direction $\mathbf{N} \times \mathbf{E}_{\Theta}^{\prime}$, but we note that $\mathbf{E}_{Z}^{\prime}$ cannot be defined as $\mathcal{P} \mathbf{E}_{Z} /\left|\mathcal{P} \mathbf{E}_{Z}\right|$ since this is not expressible as $\mathbf{R E}_{Z}$ and is not perpendicular to $\mathbf{E}_{\Theta}^{\prime}$. The form of $\mathbf{R}$ is determined numerically from the geometry of the computational model of the AAA.

\section{Results}

In order to illustrate the theory of the preceding sections we now summarize the numerical results of a nonlinear finite element analysis that uses the patient specific AAA geometry and patient based material parameters described above. The numerical procedure makes use of a purposely written material subroutine, called UMAT, based on the constitutive formulations given in (66) and (67), and uses Abaqus to solve the corresponding equations.

In many previous papers, exemplified by Raghavan and Vorp (2000), Raghavan et al. (2000), Wang et al. (2002), Lu et al. (2007), Scotti et al. (2008) and Maier et al. (2010), the von Mises stress has been used to characterize the stress distributions in the walls of AAAs, and here we also adopt this as our stress measure, which we denote by $\sigma_{\mathrm{vm}}$, defined as $\sigma_{\mathrm{vm}}=\sqrt{3 \overline{\boldsymbol{\sigma}}: \overline{\boldsymbol{\sigma}} / 2}$. The wall stress distribution on the inner and outer surfaces of the AAA wall is then visualized on the geometry of the reference configuration $\mathcal{B}_{\mathrm{r}}$. Our computation has shown that when the residual stress is included in the model the magnitudes and distributions of the von Mises stress are very similar to those without residual stress, and so we illustrate only the former. 
The results of our computation, based on the model energy function with residual stress included, are shown in Figs. A.4 and A.5. First, the von Mises stress distribution in a AAA at a physiological relevant pressure applied to the inner surface is shown in Fig. A.4. The two images in Fig. A.4(a) and (b) show the distribution on the inner surface, right and left sides respectively, and (c) and (d) provide the distribution on the outer surface of the AAA, left and right sides respectively. For ease of reference the orientation of the global Cartesian coordinate system $(X, Z)$ in the plane section dividing the two sections of the AAA is included in each of the images. Note, however, that there is no plane of symmetry in the AAA and the shapes of the right and left sections are slightly different.

As expected, the magnitude of the stress is non-uniform and varies widely over each of the inner and outer surfaces. Not surprisingly, the magnitude on the inner surface is, in general, larger than that on the outer surface. In particular, a maximum value of the von Mises stress of $460.85 \mathrm{kPa}$ was found on the inner surface of the left side of the AAA wall shown in Fig. 4(b).

Secondly, the magnitude of the displacement $|\mathbf{u}|$ distributed on the inner and outer surfaces is shown in the four images in Fig. A.5. Similarly to the von Mises stress distribution, the displacement results with and without residual stress are very similar and therefore only those including the residual stress are shown. The maximum displacement for an applied physiological pressure is given by $|\mathbf{u}|=16.89 \mathrm{~mm}$. For the computation in which the residual stress is not included the magnitude is similar and to within \pm 0.03 $\mathrm{mm}$ at the each location.

The variation of the von Mises stress across the wall thickness is presented in Figs. A.6 and A.7. In Fig. A.6 this is provided at the location where the von Mises stress on the inner surface assumes its maximum value. The discontinuous gradient arises because Abaqus calculates the stress values at the integration point of each element and then extrapolates these values to the nodal points. Since there are four elements through the wall thickness there are five corresponding nodal points, and the values of the stresses at these points have been connected by straight lines. Clearly, the value of the von Mises stress drops off significantly from the inner to the outer wall. Results with and without residual stress are shown. While there is in general little difference the residual stress has the effect of reducing the von Mises stress slightly near the inner surface.

The path in Fig. A.7 is chosen at a location where the values of the stress are smaller but larger on the outer surface than on the inner surface. In this 
case the residual stress reduces the von Mises stress near the inner surface but increases it near the outer surface more significantly than illustrated in Fig. A.6. There is a gradient discontinuity at only one nodal point.

\section{Concluding discussion}

In this paper we have developed a general constitutive formulation of nonlinear elasticity incorporating two preferred directions and a general residual stress, with the energy function dependent on invariants of the right CauchyGreen tensor, the preferred direction vectors and the residual stress tensor.

The constitutive equation used to validate and verify the analytical aspect of the work is representative of an anisotropic material characterized by two preferred directions and the inclusion of residual stress. For clarity of presentation, we decided on a formulation most frequently used in modeling soft anisotropic materials. Constitutive laws that account for distributed fiber orientations (Lanir, 1983; Gasser et al., 2006; Ferruzzi et al., 2011; Gasser et al., 2012) and for multi-scale modeling (Martufi and Gasser, 2011) are available. However, open questions are knowledge of the spatial variation of mechanical properties, of residual stress distribution and of collagen fiber orientations (Saini et al., 1995). In vivo characterization of the entire AAA wall is necessary to obtain regional variation of mechanical properties to accurately determine the unloaded configuration and ultimately a real patient specific wall stress distribution.

For numerical purposes, the energy function has been decomposed into isochoric and volumetric parts. This has then been particularized so that the isochoric part consists of an exponential isotropic term associated with the matrix material within which the preferred directions (fibre orientations) are embedded, two anisotropic exponential terms associated with these directions, and a simple additional term reflecting the presence of residual stress. The volumetric part consists of a penalty function for enforcing the incompressibility constraint and a term dependent on the residual stress to ensure that conditions that the energy function must satisfy in the reference configuration are met.

An elasticity tensor associated with this formulation and based on the Jaumann derivative of the Kirchhoff stress is then obtained for purposes of numerical implementation using Abaqus.

There are no reliable data on residual stresses in AAAs, and, therefore, for this study we consider the residual stresses to be based on the expressions 
suggested in Merodio et al. (2013) and Merodio and Ogden (2015) for a circular cylindrical tube. These are consistent with the known residual stress distributions arising from the opening angle method. The corresponding model parameters have been selected to obtain residual stress magnitudes similar to those given in Ogden (2003); Holzapfel and Ogden (2010) and Alford et al. (2010).

To represent the fibre directions and the residual stress in the AAA a new method has been proposed that maps the two fibre orientations and the residual stress in a circular cylindrical tube configuration onto the irregular geometry of the AAA in a realistic fashion.

To illustrate the efficacy of the formulation we have summarized the main results of a finite element analysis based on a patient specific AAA geometry. It was found that when the effect of residual stress is neglected the maximum von Mises stress is $478.14 \mathrm{kPa}$, which reduces to $460.85 \mathrm{kPa}$ when the residual stress is included. This finding is consistent with the now accepted understanding that residual stresses in tissues optimize the mechanical performance by homogenizing stress levels. The papers of Vaishnav and Vossoughi (1987), Delfino et al. (1997), Holzapfel et al. (2000), Alastrué et al. (2010) and Pierce et al. (2015), for instance, report similar findings that support this understanding. However, the trend shown in Fig. A.7 indicates that the opposite effect is possible, and this arises because of the irregular shape of the AAA associated with the pathology, which seems to counteract the homogenization that would be expected in a healthy tissue.

It is true that, in general, the residual stress field does have a significant influence on the transmural stress gradient. However, as pointed out by Labrosse et al. (2013), it is not true that residual stress always reduces the stress gradient across the arterial wall. Labrosse et al. (2013) show that for an opening angle less than $90^{\circ}$, the in vivo stresses are reduced compared to the case without residual stress. However, the stress gradient keeps increasing with the opening angle. Specifically, by $160^{\circ}$ for the circumferential direction, and $120^{\circ}$ for the longitudinal direction, the gradients exceed those in the case without residual stress. We also like to recall a conclusion by Saini et al. (1995) 'These studies show that measurements of opening angle in otherwise intact rings cannot reveal true values of residual strains'. Hence, they provide an indication of the magnitude of residual stress.

The inclusion of residual stress lowers the magnitude of the maximum stress, our results show that this effect is quite small. By contrast, other authors, including Delfino et al. (1997), Holzapfel et al. (2000), Alastrué et 
al. (2010) and Pierce et al. (2015), for various arteries, report significant reductions in stress levels when residual stress is accounted for. In particular, Pierce et al. (2015) applied their analysis to AAAs, but in the absence of data they suggested that they may have overestimated the magnitudes of the residual stresses. For the simulations in this paper, the wall thickness to diameter ratio is much smaller than that reported by Delfino et al. (1997), Alastrué et al. (2010), which is consistent with the smaller effect of the residual stress reported here. As is the case for the von Mises stress the magnitudes of the displacement field are only slightly modified when residual stresses are included in the model.

We emphasize that we have presented a preliminary analysis here based on inclusion of residual stress in a very simple way. However, the general formulation that we have provided will allow adoption of more complex models within the same framework when this can be justified by the availability of detailed data on residual stresses in AAAs. Clearly, it is important to obtain more data to properly inform the modelling process.

\section{Acknowledgement}

This research was supported by the National Science Foundation [Grant number CMMI-1031366] and by a Faculty Research Award provided by Tufts University.

\section{Appendix A. A version of the Cayley-Hamilton theorem}

Following Rivlin (1955) we note that for two second-order tensors $\mathbf{C}$ and $\tau$ in three dimensions the Cayley-Hamilton theorem leads to

$$
\begin{aligned}
& \mathbf{C}^{2} \boldsymbol{\tau}+\mathbf{C} \boldsymbol{\tau} \mathbf{C}+\boldsymbol{\tau} \mathbf{C}^{2}-I_{1}(\mathbf{C})(\mathbf{C} \boldsymbol{\tau}+\boldsymbol{\tau} \mathbf{C})-I_{1}(\boldsymbol{\tau}) \mathbf{C}^{2}+I_{2}(\mathbf{C}) \boldsymbol{\tau} \\
+ & {\left[I_{1}(\mathbf{C}) I_{1}(\boldsymbol{\tau})-\operatorname{tr}(\mathbf{C} \boldsymbol{\tau})\right] \mathbf{C}-\left[\operatorname{tr}\left(\mathbf{C}^{2} \boldsymbol{\tau}\right)-I_{1}(\mathbf{C}) \operatorname{tr}(\mathbf{C} \boldsymbol{\tau})+I_{2}(\mathbf{C}) I_{1}(\boldsymbol{\tau})\right] \mathbf{I}_{\mathrm{r}}=\mathbf{O}, }
\end{aligned}
$$

where $I_{1}$ and $I_{2}$ are the principal invariants of their indicated arguments, and $\mathbf{O}$ is the null tensor. Note that this applies whether or not $\mathbf{C}$ and $\boldsymbol{\tau}$ are symmetric, and a corresponding identity holds when $\mathbf{C}$ and $\boldsymbol{\tau}$ are interchanged.

This can be used as a basis for obtaining connections between different invariants. We give three examples. By taking $\boldsymbol{\tau}=\mathbf{M} \otimes \mathbf{M}^{\prime}$ and then 
contracting (A.1) with $\mathbf{M}^{\prime} \otimes \mathbf{M}$ we obtain

$$
\begin{aligned}
2\left(\mathbf{M} \cdot \mathbf{M}^{\prime}\right)\left[\left(\mathbf{C}^{2} \mathbf{M}\right) \cdot \mathbf{M}^{\prime}\right] & =I_{2}\left[1-\left(\mathbf{M} \cdot \mathbf{M}^{\prime}\right)^{2}\right]+I_{5}+I_{7}+I_{4} I_{6} \\
& -I_{1}\left(I_{4}+I_{6}\right)+2\left(\mathbf{M} \cdot \mathbf{M}^{\prime}\right) I_{1} I_{8}-I_{8}^{2},
\end{aligned}
$$

which shows that $\left(\mathbf{C}^{2} \mathbf{M}\right) \cdot \mathbf{M}^{\prime}$ can be expressed in terms of the invariants $I_{1}, I_{2}, I_{4}, I_{5}, I_{6}, I_{7}, I_{8}$ of $\mathbf{C}$, where here $I_{8}$ is defined as $\mathbf{M} \cdot\left(\mathbf{C M}^{\prime}\right)$. When $\mathbf{M} \cdot \mathbf{M}^{\prime}=0$ this reduces to the connection

$$
I_{8}^{2}=I_{2}+I_{4} I_{6}+I_{5}+I_{7}-I_{1}\left(I_{4}+I_{6}\right)
$$

noted in (6).

For the second example (A.1) is contracted with $\mathbf{M} \otimes \mathbf{M}^{\prime}$, which shows that $\mathbf{M} \cdot\left(\mathbf{C} \boldsymbol{\tau} \mathbf{C M}^{\prime}\right)$ can be expressed in terms of the invariants listed in (16) and earlier equations. Similarly, by replacing $\boldsymbol{\tau}$ by $\boldsymbol{\tau}^{2}$ in (A.1) it follows that $\mathbf{M} \cdot\left(\mathbf{C} \boldsymbol{\tau}^{2} \mathbf{C M}^{\prime}\right)$ can be expressed in terms of the invariants (17) and those listed in earlier equations.

For the third example, we give an expression for $\mathbf{M} \cdot\left(\mathbf{C}^{2} \boldsymbol{\tau} \mathbf{C}^{2} \mathbf{M}^{\prime}\right)$. By multiplying (A.1) on the right by $\mathbf{C}^{2}$ and rearranging the result with the help of the standard Cayley-Hamilton theorem

$$
\mathbf{C}^{3}-I_{1} \mathbf{C}^{2}+I_{2} \mathbf{C}-I_{3} \mathbf{I}_{\mathrm{r}}=\mathbf{O},
$$

and then contracting with $\mathbf{M} \otimes \mathbf{M}^{\prime}$, we obtain

$$
\begin{aligned}
\mathbf{M} \cdot\left(\mathbf{C}^{2} \boldsymbol{\tau} \mathbf{C}^{2} \mathbf{M}^{\prime}\right) & =I_{2}(\mathbf{C}) \mathbf{M} \cdot\left(\mathbf{C} \boldsymbol{\tau} \mathbf{C M}^{\prime}\right)-I_{3}(\mathbf{C})\left[\mathbf{M} \cdot\left(\mathbf{C} \boldsymbol{\tau} \mathbf{M}^{\prime}\right)+\mathbf{M} \cdot\left(\boldsymbol{\tau} \mathbf{C M}^{\prime}\right)\right] \\
& +\left[I_{1}(\boldsymbol{\tau}) I_{3}(\mathbf{C})-I_{2}(\mathbf{C}) \operatorname{tr}(\mathbf{C} \boldsymbol{\tau})\right] \mathbf{M} \cdot\left(\mathbf{C} \mathbf{M}^{\prime}\right) \\
& +\operatorname{tr}\left(\mathbf{C}^{2} \boldsymbol{\tau}\right) \mathbf{M} \cdot\left(\mathbf{C}^{2} \mathbf{M}^{\prime}\right)+I_{3}(\mathbf{C}) \operatorname{tr}(\mathbf{C} \boldsymbol{\tau}) \mathbf{M} \cdot \mathbf{M}^{\prime}
\end{aligned}
$$

This shows that $\mathbf{M} \cdot\left(\mathbf{C}^{2} \boldsymbol{\tau} \mathbf{C}^{2} \mathbf{M}^{\prime}\right)$ can be expressed in terms of the previously listed invariants.

This procedure can be followed by variously pre- and post-multiplying (A.1) by $\mathbf{C} \boldsymbol{\tau}$ or $\boldsymbol{\tau} \mathbf{C}$, or by replacing $\boldsymbol{\tau}$ by, for example, $\boldsymbol{\tau} \mathbf{C} \boldsymbol{\tau}$ and similar combinations and then contracting the results with $\mathbf{M} \otimes \mathbf{M}^{\prime}$ to obtain further connections between expressions of the form $\mathbf{M} \cdot\left(\mathbf{C}^{\alpha} \boldsymbol{\tau}^{\beta} \mathbf{C}^{\gamma} \boldsymbol{\tau}^{\delta} \ldots \mathbf{M}^{\prime}\right)$, where $\alpha, \beta, \gamma, \delta \cdots \in\{0,1,2\}$.

\section{References}

Abaqus, 2013. Theory Manual. Dassault Systèmes Simulia, Providence, RI, USA. 
Adler, J.H., Dorfmann, L., Han, D., MacLachlan, S., Paetsch, C., 2014. Mathematical and computational models of incompressible materials subject to shear. IMA J. Appl. Math. 79, 889-914.

Alastrué, V., Peña, E., Ángel, M., Martínez, M.A., Doblaré, M., 2007. Assessing the use of the opening angle method to enforce residual stresses in patient-specific arteries. Ann. Biomed. Eng. 35, 1821-1837.

Alastrué, V., Garía, A., Peña, E., Rodríguez, J.F., Martínez, M.A., Doblaré, M., 2010. Numerical framework for patient-specific computational modelling of vascular tissue. Int. J. Numer. Meth. Biomed. Engng. 26, 35-51.

Alford, P.W., Feinberg, A.W., Sheehy, S.P., Parker, K.K., 2010. Biohybrid thin films for measuring contractility in engineered cardiovascular muscle. Biomaterials 31, 3613-3621.

Alford, P.W., Humphrey, J.D., Taber, L.A., 2008. Growth and remodeling in a thick-walled artery model: Effects of spatial variations in wall constituents. Biomech. Model. Mechanobiol. 7, 245-262.

Ambrosi, D., Guillou, A., Di Martino, E.S., 2008. Stress-modulated remodeling of a non-homogeneous body. Biomech. Model. Mechanobiol. 7, 63-76.

Balzani, D., Schröder, J., Gross, D., 2008. Numerical simulation of residual stresses in arterial walls. Comp. Mater. Sci. 39, 117-123.

Chuong, C.J., Fung, Y.C., 1986. On residual stresses in arteries. J. Biomech. Eng. 108, 189-192.

Costa, K.D., May-Newman, K., Farr, D., O’Dell, W.G., McCulloch, A.D., Omens, J.H., 1997. Three-dimensional residual strain in midanterior canine left ventricle. Am. J. Physiol. 273, H1968-H1976.

Delfino, A., Stergiopulos, N., Moore, J.E., Meister, J.J., 1997. Residual strain effects on the stress field in a thick wall finite element model of the human carotid bifurcation. J. Biomech. 30, 777-786.

Demiray, H., 1972. Elasticity of soft biological tissues. J. Biomech. 5, 309311. 
Dorfmann, A., Wilson, C., Edgar, E.S., Peattie, R.A., 2010. Evaluating patient-specific abdominal aortic aneurysm wall stress based on flowinduced loading. Biomech. Model. Mechanobiol. 9, 127-139.

Ferruzzi, J., Vorp, D.A., Humphrey J.D., 2011. On constitutive descriptors of the biaxial mechanical behaviour of human abdominal aorta and aneurysms. J. R. Soc. Interface 8, 435-450.

Flory, P.J., 1961. Thermodynamic relations for highly elastic materials. Trans. Faraday Soc. 57, 829-838.

Fung, Y.C., 1983. On the foundations of biomechanics. J. Appl. Mech. 50, 1003-1009.

Gasser, T.D., Ogden, R.W., Holzapfel G.A., 2006. Hyperelastic modelling of arterial layers with distributed collagen fibre orientations. J. R. Soc. Interface 3, 15-35.

Gasser, T.D., Gallinetti S., Xing, X., Forsell, C., Swedenborg, J, Roy, J., 2012. Spatial orientation of collagen fibers in the abdominal aortic aneurysms wall and its relation to wall mechanics. Acta Biomater. 8, 30913103.

Hoger, A., 1985. On the residual stress possible in an elastic body with material symmetry. Arch. Rat. Mech. Anal. 88, 271-290.

Holzapfel, G.A., 2000. Nonlinear Solid Mechanics: A Continuum Approach for Engineering. John Wiley \& Sons, Chichester.

Holzapfel, G.A., Gasser, T.C., Ogden, R.W., 2000. A new constitutive framework for arterial wall mechanics and a comparative study of material models. J. Elasticity 61, 1-48.

Holzapfel, G.A., Ogden, R.W., 2009. Constitutive modelling of passive myocardium: a structurally based framework for material characterization.Phil. Trans. R. Soc. Lond. A 367, 3445-3475.

Holzapfel, G.A., Ogden, R.W., 2010. Modelling the layer-specific threedimensional residual stresses in arteries, with an application to the human aorta. J. R. Soc. Interface 7, 787-799. 
Humphrey, J.D., Rajagopal, K.R., 2002. A constrained mixture model for growth and remodeling of soft tissues. Math. Mod. Meth. Appl. S. 12, 407-430.

Humphrey, J.D., Holzapfel, G.A., 2012. Mechanics, mechanobiology, and modeling of human abdominal aorta and aneurysms. J. Biomech. 45, 805814 .

Labrosse, M.R., Gerson, E.R., Veinot, J.P., Beller C.J., 2013. Mechanical characterization of human aortas from pressurization testing and a paradigm shift for circumferential residual stress. J. Mech. Behav. Biomed. $17,44-55$.

Lanir, Y., 1983. Constitutive equations for fibrous connective tissues. J. Biomech. 1, 1-12.

Lu, J., Zhou, X., Raghavan, M.L., 2007. Inverse elastostatic stress analysis in pre-deformed biological structures: Demonstration using abdominal aortic aneurysms. J. Biomech. 40, 693-696.

Maier, A., Gee, M.W., Reeps, C., Pongratz, J., Eckstein, H.-H., Wall, W.A., 2010. A comparison of diameter, wall stress, and rupture potential index for abdominal aortic aneurysm rupture risk prediction. Ann. Biomed. Eng. 38, 3124-3134.

Martufi, G., Gasser, T.D., 2011. A constitutive model for vascular tissue that integrates fibril, fiber and continuum levels with application to the isotropic and passive properties of the infrarenal aorta. J. Biomech. 44, $2544-2550$.

MATLAB, 2014. The MathWorks Inc. Natick, MA, USA.

Merodio, J., Ogden, R.W., 2006.The influence of the invariant $I_{8}$ on the stress-deformation and ellipticity characteristics of doubly fiber-reinforced nonlinearly elastic solids.Int. J. Non-Linear Mech. 41, 556-563.

Merodio, J., Ogden, R.W., Rodríguez, J., 2013. The influence of residual stress on finite deformation elastic response. Int. J. Non-Linear Mech. 56, 43-49. 
Merodio, J., Ogden, R.W., 2015. Extension, inflation and torsion of a residually stressed circular cylindrical tube. Continuum Mech. Thermodyn. (in press), DOI 10.1007/s00161-015-0411-z.

Nienhaus, U., Aegerter-Wilmsen, T., Aegerter, C.M., 2009. Determination of mechanical stress distribution in Drosophila wing discs using photoelasticity. Mech. Develop. 126, 942-949.

Ogden, R.W., 1978. Nearly isochoric elastic deformations: application to rubberlike solids. J. Mech. Phys. Solids 26, 37-57.

Ogden, R.W., 1997. Non-Linear Elastic Deformations. Dover Publications.

Ogden, R.W., 2003. Nonlinear elasticity, anisotropy and residual stresses in soft tissue. In: Holzapfel, G.A., Ogden, R.W. (Eds.), Biomechanics of Soft Tissue in Cardiovascular Systems, CISM Courses and Lectures Series no. 441. Springer, Wien, pp. 65-108.

Ogden, R.W., 2015. Nonlinear elasticity with application to soft fibrereinforced materials. In: Dorfmann, L., Ogden, R.W. (Eds.), Nonlinear Mechanics of Soft Fibrous Materials, CISM Courses and Lectures Series no. 559. Springer, Wien, pp. 1-48.

Olsson, T., Klarbring, A., 2008. Residual stresses in soft tissue as a consequence of growth and remodeling: application to an arterial geometry. Eur. J. Mech. A-Solids 27, 959-974.

Pancheri, F.Q., 2014. Experimental and analytical aspects of biological and engineering materials subject to planar biaxial loading. Ph.D. thesis, Tufts University.

Penn, R.W., 1970. Volume changes accompanying the extension of rubber. Trans. Soc. Rheol. 14, 509-517.

Pierce, D.M., Fastl, T.E., Rodriguez-Vila, B., Verbrugghe, P., Fourneau, I., Maleux, G., Herijgers, P., Gomez, E.J., Holzapfel, G.A., 2015. A method for incorporating three-dimensional residual stretches/stresses into patientspecific finite element simulations of arteries. J. Mech. Behav. Biomed. Mater. 47, 147-164. 
Polzer, S., Bursa, J., Gasser, T.C., Staffa, R., Vlachovsky, R., 2013. A numerical implementation to predict residual strains from the homogeneous stress hypothesis with application to abdominal aortic aneurysms. Ann. Biomed. Eng. 41, 1516-1527.

Raghavan, M.L., Vorp, D.A., 2000. Toward a biomechanical tool to evaluate rupture potential of abdominal aortic aneurysm: identification of a finite strain constitutive model and evaluation of its applicability. J. Biomech. $33,475-482$.

Raghavan, M.L., Vorp, D.A., Federle, M.P., Makaroun, M.S., Webster, M.W., 2000. Wall stress distribution on three-dimensionally reconstructed models of human abdominal aortic aneurysm. J. Vasc. Surg. 31, 760-769.

Rivlin, R.S., 1955. Further remarks on the stress-deformation relations for isotropic materials. J. Rat. Mech. Anal. 4, 681-702.

Saini, A., Berry, C., Greenwald, S. 1995. Effect of age and sex on residual stress in the aorta. J. Vasc. Res. 32, 398-405.

Scotti, C.M., Jimenez, J., Muluk, S.C., Finol, E.A., 2008. Wall stress and flow dynamics in abdominal aortic aneurysms: finite element analysis vs. fluid-structure interaction. Comp. Meth. Biomech. Biomed. Eng. 11, 301322 .

Shams, M., Destrade, M., Ogden, R.W., 2011. Initial stresses in elastic solids: Constitutive laws and acoustoelasticity. Wave Motion 48, 552-567.

Skalak, R., Zargaryan, S., Jain, R.K., Netti, P.A., Hoger, A., 1996. Compatibility and the genesis of residual stress by volumetric growth. J. Math. Biol. 34, 889-914.

Spencer, A.J.M., 1971. Theory of Invariants. In: Eringen, A.C. (Ed.), Continuum Physics. Vol. 1. Academic Press, New York, pp. 239-353.

Spencer, A.J.M., Rivlin, R.S., 1959. Finite integrity bases for five or fewer symmetric $3 \times 3$ matrices. Arch. Rat. Mech. Anal. 2, 435-446.

Taber, L.A., 1995. Biomechanics of growth, remodeling and morphogenesis. App. Mech. Rev. 48, 487-545. 
Tierney, A.P., Dumont, D.M., Callanan, A., Trahey, G.E., McGloughlin, T.M., 2010. Acoustic radiation force impulse imaging on ex vivo abdominal aortic aneurysm model. Ultrasound Med. Biol. 36, 821-832.

Vaishnav, R.N., Vossoughi, J., 1987. Residual stress and strain in aortic segments. J. Biomech. 20, 235-239.

Vandiver, R., Goriely, A., 2009. Differential growth and residual stress in cylindrical elastic structures. Phil. Trans. R. Soc. Lond. A 367, 3607-3630.

Wang, D.H.J., Makaroun, M.S., Webster, M.W., Vorp, D.A., 2002. Effect of intraluminal thrombus on wall stress in patient-specific models of abdominal aortic aneurysm. J. Vasc. Surg. 36, 598-604.

Wang, H.M., Luo, X.Y., Gao, H., Ogden, R.W., Griffith, B.E., Berry, C., Wang, T.J., 2014. A modified Holzapfel-Ogden law for a residually stressed finite strain model of the human left ventricle in diastole. Biomech. Model. Mechanobiol. 13, 99-113.

Wang, R., Raykin, J., Gleason, R.L., Ethier, C.R., 2015. Residual deformations in ocular tissues. J. R. Soc. Interface 12, 20141101.

Wells, S.M., Walter, E.J., 2010. Changes in the mechanical properties and residual strain of elastic tissue in the developing fetal aorta. Ann. Biomed. Eng. 38, 345-356.

Yeni, Y.N., Schaffler, M.B., Gibson, G., Fyhrie, D.P., 2002. Prestress due to dimensional changes caused by demineralization: A potential mechanism for microcracking in bone. Ann. Biomed. Eng. 30, 217-225. 


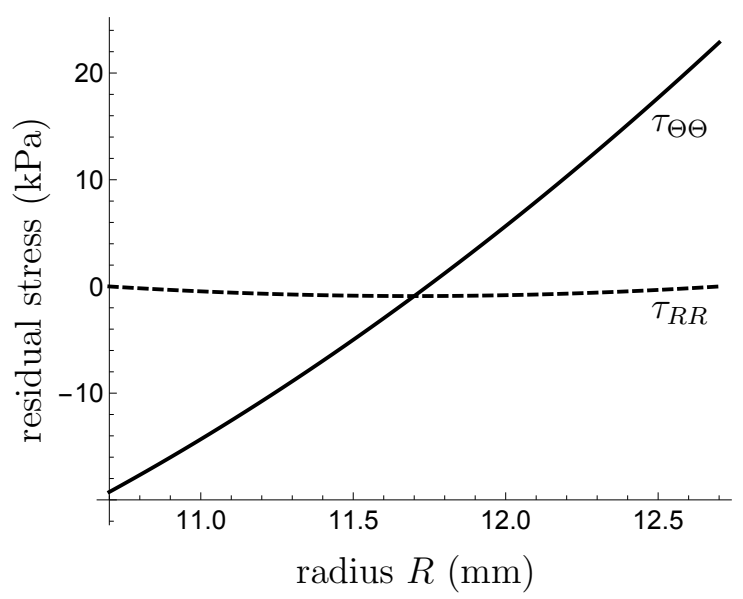

Figure A.1: Distribution of the residual stress components $\tau_{R R}$ and $\tau_{\Theta \Theta}$ as a function of the radius $R$. The radial component satisfies the boundary conditions $\tau_{R R}=0$ on the inner and outer surfaces located at $R=10.7$ and $12.7 \mathrm{~mm}$, respectively. The azimuthal component $\tau_{\Theta \Theta}$ is compressive on the inner and tensile on the outer surface.

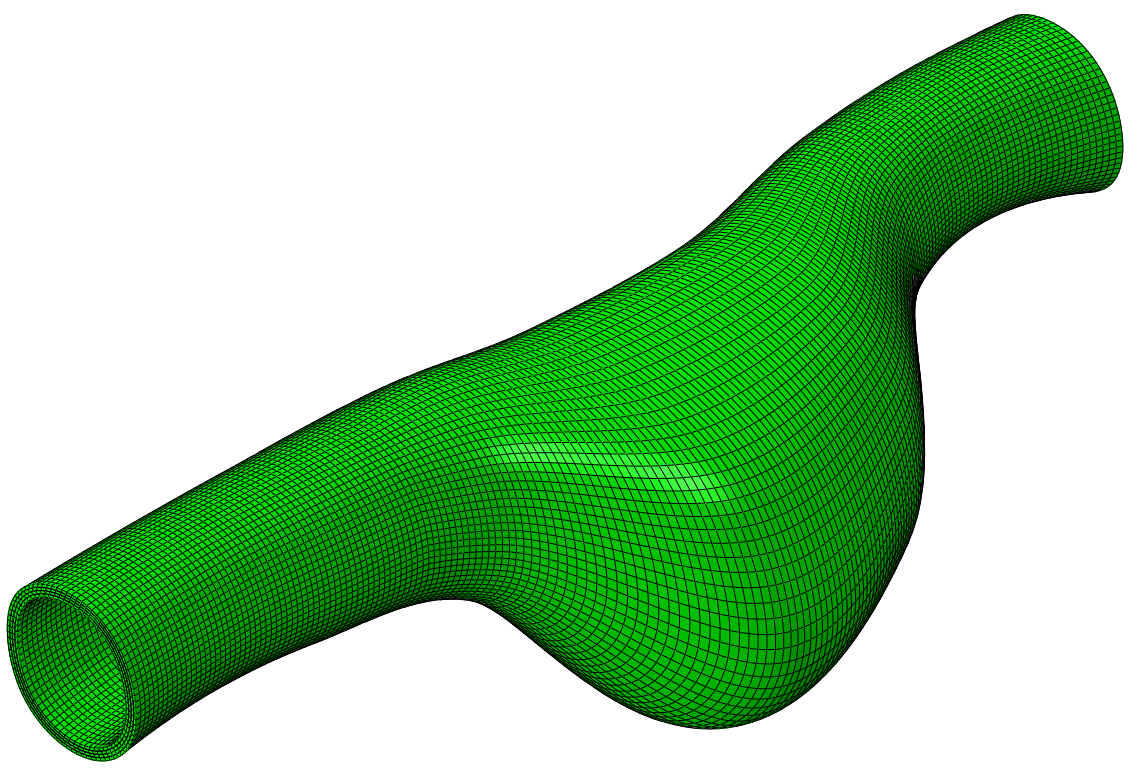

Figure A.2: Geometric layout and element distribution of the AAA model. Four hexahedral elements are used through the wall thickness. 
Figure A.3: Location of the piecewise linear centre line, which is used to define a local cylindrical coordinate system with unit basis vectors $\mathbf{E}_{R}, \mathbf{E}_{\Theta}, \mathbf{E}_{Z}$.

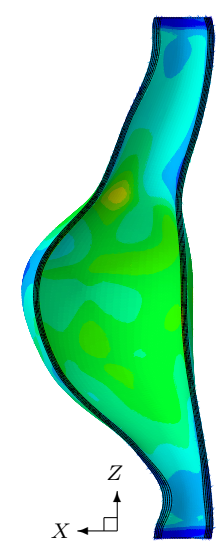

(a)

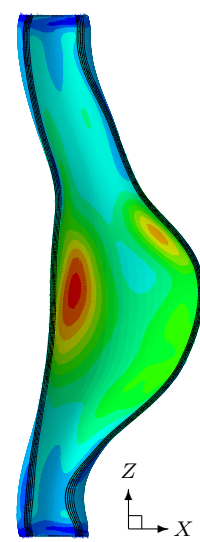

(b)

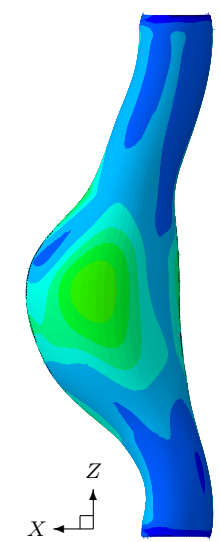

(c)

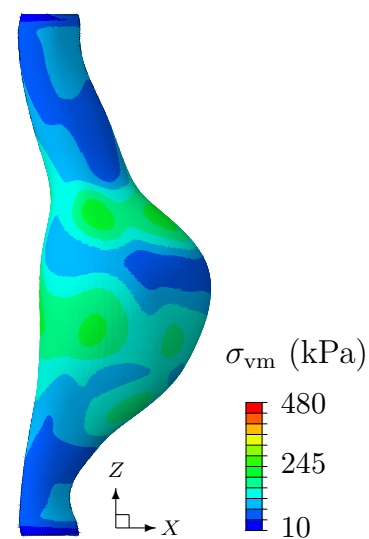

(d)

Figure A.4: The von Mises stress distribution on the inner surfaces of the AAA when a physiological relevant pressure is applied is shown mapped on to the reference configuration of the AAA in (a) and (b), respectively the right and left sides of the AAA. The corresponding distributions on the outer surface are shown in (c) and (d), left and right sides, respectively. The pressure applied to the inner surface is uniform in the circumferential direction but varies longitudinally. 


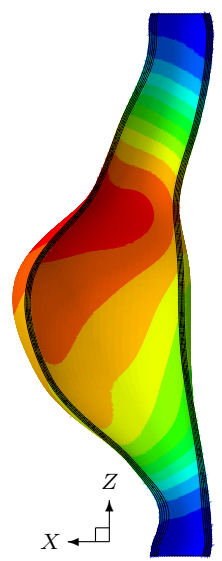

(a)

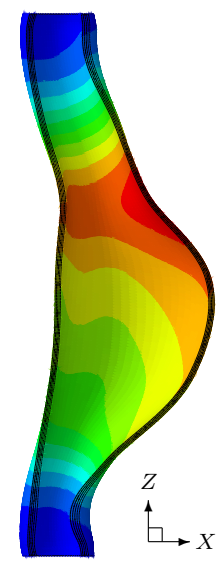

(b)

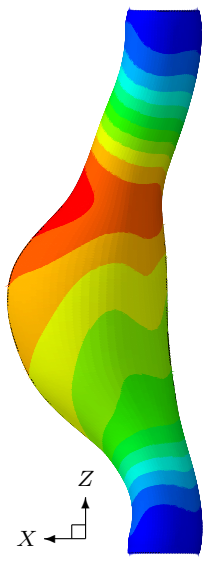

(c)

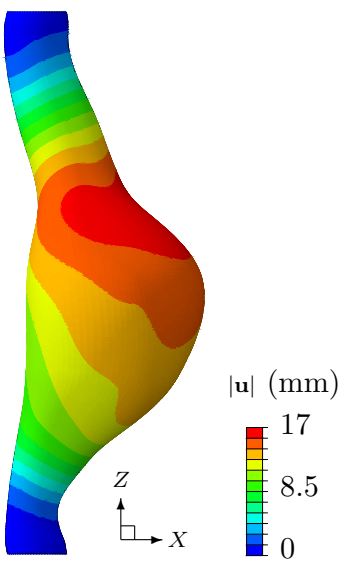

(d)

Figure A.5: The magnitude $|\mathbf{u}|$ of the displacement is shown mapped on to the reference configuration of the AAA subject to an internal pressure. (a) and (b), respectively, show values on the inner surface corresponding to the right and left sides of the AAA, while (c) and (d) show corresponding results for the outer surface, left and right sides, respectively.

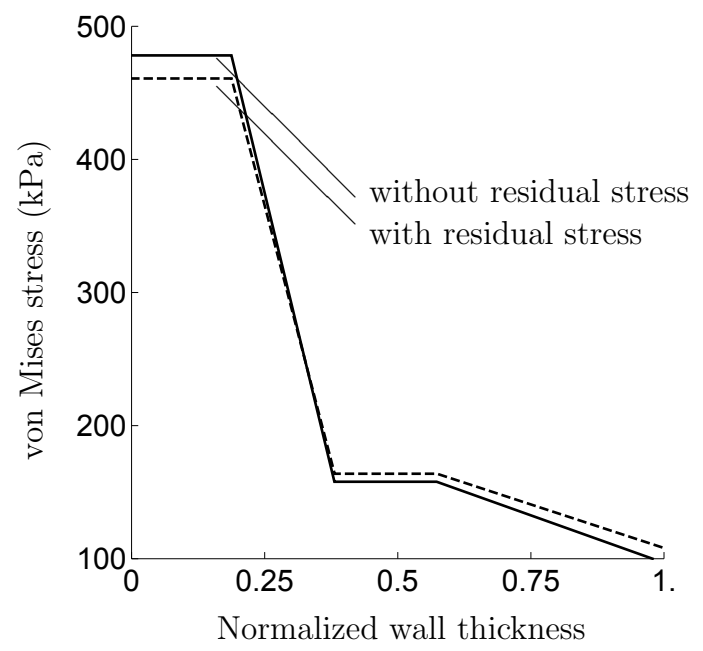

Figure A.6: Variation of the von Mises stress across the wall thickness at the location where the von Mises stress on the inner surface assumes its maximum value. Solid (dashed) lines indicate values without (with) residual stress included. 
Figure A.7: Variation of the von Mises stress across the wall thickness at a location where the stress increases from the inner to the outer surface. Solid (dashed) lines indicate values without (with) residual stress included.

Table A.1: Patient specific values of the parameters in the isochoric and volumetric parts of the energy function (66) and (67). The values of $\mu, \mu_{\mathrm{f}}$ and $\kappa$ are given in $\mathrm{kPa}$, the angle $\varphi$ in degrees.

\begin{tabular}{|c|c|c|c|c|c|}
\hline \multicolumn{6}{|c|}{ Summary of model parameters } \\
\hline$\mu(\mathrm{kPa})$ & $\alpha$ & $\mu_{\mathrm{f}}(\mathrm{kPa})$ & $k$ & $\varphi^{\circ}$ & $\kappa(\mathrm{kPa})$ \\
\hline 46.987 & 21.071 & 33.922 & 37.287 & 6.7 & 20,000 \\
\hline
\end{tabular}

